\title{
CS Research Square

\section{Integrative analysis of outer membrane vesicles proteomics and whole-cell transcriptome analysis of eravacycline induced Acinetobacter baumannii strains}

\section{Dinesh Kumar Kesavan}

Jiangsu University

Aparna Vasudevan

Jiangsu University

Liang Wu

Jiangsu University

Jianguo Chen

the affiliated people's hospital, jiangsu university, zhenjiang

\section{Zhaoliang Su}

Jiangsu University

\section{Shengjun Wang}

Jiangsu University

Huaxi Xu ( $\nabla$ xuhx@ujs.edu.cn)

Jiangsu University https://orcid.org/0000-0002-2568-7393

Research article

Keywords: Acinetobacter baumannii, Eravacycline, Outer membrane vesicles, Whole-cell transcriptome, OMVs proteome

Posted Date: October 2nd, 2019

DOI: https://doi.org/10.21203/rs.2.15542/v1

License: (9) This work is licensed under a Creative Commons Attribution 4.0 International License.

Read Full License

Version of Record: A version of this preprint was published at BMC Microbiology on February 11th, 2020. See the published version at https://doi.org/10.1186/s12866-020-1722-1. 


\section{Abstract}

Background Acinetobacter baumannii is a multidrug resistant (MDR) hazardous bacterium with very high antimicrobial resistance profiles. Outer membrane vesicles (OMVs) help directly and/or indirectly towards antibiotic resistance in these organisms. The present study aims to look on the proteomic profile of OMV as well as on the bacterial transcriptome upon exposure and induction with eravacycline, a new synthetic fluorocycline. RNA sequencing analysis of whole cell and LC-MS/MS proteomic profiling of OMV proteome abundance were done to identify the differential expression among the eravacycline-induced $A$. baumannii ATCC 19606 and A. baumannii clinical strain JU0126. Results The Differential expressed genes from the RNA sequencing and were analysed using R package and bioinformatics software and tools. Genes encoding drug efflux and membrane transport were upregulated among the DEGs from both ATCC 19606 and JU0126 strains. As evident with the induction of eravacycline resistance, ribosomal proteins were upregulated in both the strains in the transcriptome profiles and also resistance pumps, such as MFS, RND, MATE and ABC transporters. High expression of stress and survival proteins were predominant in the OMVs proteome with ribosomal proteins, chaperons, OMPs OmpA, Omp38 upregulated in ATCC 19606 strain and ribosomal proteins, toluene tolerance protein, siderophore receptor and peptidases in the JU0126 strain. The induction of resistance to eravacycline was supported by the presence of upregulation of ribosomal proteins, resistance conferring factors and stress proteins in both the strains of A. baumannii ATCC 19606 and JU0126, with the whole-cell gene transcriptome towards both resistance and stress genes while the OMVs proteome enriched more with survival proteins.Conclusion The induction of resistance to eravacycline in the strains were evident with the increased expression of ribosomal and transcription related genes/proteins. Apart from this resistance conferring efflux pumps, outer membrane proteins and stress related proteins were also an essential part of the upregulated DEGs. However, the expression profiles of OMVs proteome in the study was independent with respect to the whole cell RNA expression profiles with low to no correlation. This indicates the possible role of OMVs to be more of back-up additional protection to the existing bacterial cell defence during the antibacterial stress.

\section{Background}

Multidrug resistance (MDR) Acinetobacter baumannii is one of the most dangerous bacteria encountered among hospitalized and critically ill patients, particularly infecting the immunosuppressed patients, who undergo invasive procedures and are treated with broad-spectrum antibiotics [1]. Infections, such as ventilator-associated pneumonia (VAP), urinary tract infections, bacteremia, complicated skin and soft tissue, abdominal and central nervous system infections are commonly caused by $A$. baumannii [2]. A. baumannii strains displaying MDR properties have increased significantly in the last decades [3]. A. baumannii species have an extensive capability of antimicrobial resistance in nature owing to their impermeable outer membranes and their environmental exposure to large reservoir of resistance genes [4]. The presence of wide range of resistance genes in A. baumannii succors easy evolution from the stress of antibiotics, making them extremely difficult in elimination. Some strains are also resistance to 
polymyxins-peptides making infected patient treatment more complicated and also impossible in some cases leading to fatality $[5,6]$.

Tigecycline is the first identified glycycyline antibiotic, belonging to the tetracycline class of antibiotics that is used as the last resort antibiotic for the treatment of MDR A. baumannii [7]. Eravacycline is a broad-spectrum synthetic fluorocycline with novel c-9 pyrrolidinoacetamido and c-7 fluoro modifications. Eravacycline is also successfully used against MDR strains in case of serious infections [8]. Reports have claimed that eravacycline showed broad-spectrum activity against most bacterial resistant pathogens with $\mathrm{MIC}_{90}$ values ranging from $\leq 0.008$ to $2 \mu \mathrm{g} / \mathrm{mL}$, except $P$. aeruginosa and Burkholderia cenocepacia $\left(\mathrm{MIC}_{90}\right.$ values of $\left.16-32 \mu \mathrm{g} / \mathrm{mL}\right)[9,10]$. In studies from the New York City Hospitals on 4000 contemporary Gram-negative pathogens, eravacycline $\mathrm{MIC}_{50 / 90}$ values $(\mu \mathrm{g} / \mathrm{mL})$ for $E$. coli-K.

pneumoniae, Enterobacter aerogenes, E. cloacae and A. baumannii were 0.12/0.5, 0.25/1, 0.25/1, 0.5/1 and $0.5 / 1$ respectively [11]. Eravacycline showed good activity against MDR strains expressing extendedspectrum $\beta$-lactamases, carbapenem resistance and other types of antibiotic resistance mechanisms in Enterobacteriaceae and A. baumannii [12].

MDR bacteria have developed mechanisms to combat antibiotic stress by changing a particular metabolic process. This has been a focus of research interest with many reports based on the expression analysis in bacterium upon antibiotic exposures [13]. Outer membrane vesicles (OMVs) are mainly seen among Gram-negative organisms helping them with cell to cell communication, secretion, pathogenesis, acquisition of nutrients, self-defense and antibiotic resistance [14]. The effective contribution of OMVs towards antibiotic resistance in bacteria makes it a very important tool in the research to combat drug resistance. To date; however, there are no elaborate studies in the area of proteomic analysis with a special focus on the proteins of the OMVs from A. baumannii upon non-natural eravacycline resistance induction. By studying the proteomic profile involved with OMVs, it could be possible to identify differential expressions of proteins which are related to response to antibiotic exposure. This can be further taken to the level of metabolic pathways involved with these proteins; thereby, possibly opening new avenues identifying drug targets or drugs. [15] had used a similar approach to study OMVs proteomics in imipenem treated clinical strain of A. baumannii. In this study, we used this resource to perform proteogenomic analysis of protein components of OMVs following eravacycline treatment.

In the present study, a comparative OMVs LC-MS/MS proteome analysis and whole-cell RNA sequencebased transcriptome analysis was done with both eravacycline induced (treated) and uninduced (control) A. baumannii strains.

\section{Results}

\section{The evident increase in MIC of eravacycline upon induction of resistance}

Acinetobacter baumannii strains were exposed to sequential passages of increasing concentration of eravacycline for evaluating the acquisition of resistance. The strains with acquired resistance were 
evaluated for gene expression profiling, and OMV proteome analysis was studied to identify a specific pattern in OMVs pertaining to resistance. The MICs of eravacycline for the A. baumannii ATCC 19606 and JU0126 strain were 0.125 and $0.5 \mu \mathrm{g} / \mathrm{mL}$, respectively. The serial passage-based induction of acquired resistance among the isolates was carried out from one-eighth MIC values and above, until a lethal concentration was reached above the MIC concentration. A. baumannii ATCC 19606 was able to resist the passages up to concentration of $64 \times$ MIC after which it failed to overcome the action of eravacycline. A. baumannii JU0126 strain tolerated upon induction till concentrations of $64 \times$ the MIC of eravacycline, above which turned to be lethal concentration. Both organisms were able to present a resistant phenotype below $128 \times$ MIC concentration of eravacycline.

\section{High-throughput RNA sequence analysis}

RNA sequence analysis was performed for the A. baumannii strains, ATCC 19606 and JU0126, respectively. Both strains were grown under $64 \times$ MIC concentrations of eravacycline obtained as per induced resistance protocol described above. From high-throughput RNA sequence analysis, the length of 16205012 (an error probability of $0.03 \%$ ) and 15029752 (an error probability of $0.02 \%$ ) clean reads were obtained from eravacycline resistance induced ATCC 19606 and JU0126 strains, respectively. A total of 18732924 (an error probability of $0.03 \%$ ) and 16680372 (an error probability of $0.03 \%$ ) clean reads were obtained from control untreated strains of ATCC 19606 and JU0126 strains, respectively. The Q20 of all these four samples reached $98 \%$ for treated and $97 \%$ for control which indicated a high quality of transcriptome sequencing. The GC content (\%) were 44.03 and 43.87 (ATCC 19606 and JU0126, respectively) for treated strains and 45.11 and 45.04 (ATCC 19606 and JU0126, respectively) for control strains. Pearson's correlation between each sample was analyzed: ATCC 19606 control with ATCC 19606 treated strains, had a value of 0.774 and JU0126 control had 0.735 correlation values with treated JU0126. ATCC 19606 control strains had 0.848 correlation value with treated JU0126, ATCC 19606 control had 0.866 correlation value with control JU0126 and JU0126 control strain had 0.926 correlations with treated ATCC 19606 strains. These correlation values show high correlation between the samples.

\section{Significant DEGs among the eravacycline treated strain when compared with untreated control strains}

The complete gene expression values for A. baumannii ATCC 19606 and JU0126 eravacycline treated strains are provided in Additional file 2. In an effort to study the changes in the biological mechanisms and/or pathways of the bacterial system upon resistance to eravacycline in the treated strains when compared with the control eravacycline susceptible strains of A. baumannii ATCC 19606 and JU0126, DEGs analysis was performed (Additional file 3; Fig. 1A, B). For DEGs analysis, parameters $P$ values $(<0.05)$ and fold changes $\geq 2$ were used. A total of 1404 genes were identified as significant DEGs, of which 944 DEGs (67.2\%) were upregulated and 460 DEGs (32.8\%) were downregulated in A. baumannii ATCC 19606 treated sample when compared with control samples. From A. baumannii JU0126 strain, 1285 genes were identified as significant DEGs between each sample, of which 574 DEGs (44.7\%) were upregulated and 711 DEGs (55.4\%) were downregulated in A. baumannii JU0126 eravacycline treated strain when compared with JU0126 control strain. 


\section{GO enrichment analysis of DEGs}

$\mathrm{GO}$ enrichment is widely used to find the biological roles of each gene and its products [16]. All DEGs were mapped to their terms in GO database and compared with the reference transcriptome. GO mapped DEGs from ATCC 19606 and JU0126 were identified and classified into functional groups in three main categories: biological process, cellular process and molecular function (Fig. 1C, D). Totally, 2219 GO terms were identified in the DEGs from ATCC 19606 under all three categories; with localization, transport, cellular component, membrane, transport activity, and transmembrane transport activity being dominant terms. In JU0126, organonitrogen compound metabolism, protein metabolism, protein-containing complex, cytoplasm, structural activity and structural constituent of ribosomal were dominant terms in all three categories. Significantly enriched GO terms were considered based on the corrected $P<0.05$.

\section{Kyoto encyclopedia of genes and genomes (KEGG) analysis of DEGs}

KEGG database is a collection of various pathways, which represents the molecular interactions network between each gene/proteins [17]. To identify the enriched pathways involved in eravacycline induced strains of $A$. baumannii ATCC 19606 and clinical strain JU0126, KEGG analysis was done. In total, 78 and 86 pathways were identified in the DEGs of ATCC 19606 and JU0126 strains, respectively. In both ATCC 19606 and JU0126, microbial metabolism in diverse environments, ABC transporters, degradation of aromatic compounds and fluorobenzoate degradation pathways were the most highly represented categories. The enriched factors were represented in the ratio of the differentially expressed gene number to the total gene number in a certain pathway. The values were represented in $Q$ value, which is a corrected $P$-value ranging from 0 to 1 . The size and color gradient of the dots indicate the range between $Q$ value and the number of DEGs mapped to the indicated pathways, respectively. The top 20 values are shown in Figure 1E and 1F.

\section{Quantitative reverse transcriptase-polymerase chain reaction (qRT-PCR) validation of DEGs}

To analyze further, 6 DEGs from both upregulated and downregulated genes for both the strains were selected from the RNA sequence analysis and validated through qRT-PCR study (Fig. 2). Three genes that showed significantly increased (upregulated) expression, as well as three genes that were downregulated in each ATCC 19606 and JU0126 strains treated eravacycline compared with untreated strains, were taken further for qRT-PCR. These 6 DEGs for each strain were selected for qRT-PCR verification. From the ATCC 19606, the genes multidrug efflux RND transporter permease subunit (AU097_00445), MSF transport (AU097_00560), M1 family peptidase (AU097_00700) which upregulated with 3.2731, 1.9644, $1.2859 \log _{2}$-fold, respectively in RNA-sequencing data, showed $3.8204,2.5822$ and $2.8533 \log _{2}$-fold changes, respectively using the qRT-PCR analysis. From the RNA-sequencing data, genes porin (AU097_05635), trifunctional transcriptional regulator (AU097_15195) and transfer-RNA(AU097_11755) which downregulated with $-2.782,-1.176$ and $-3.9366 \log _{2}-$ fold, respectively, displayed-1.5859, -2.0788 and $-2.0203 \log _{2}$-fold changes, respectively using the qRT-PCR analysis. From the JU0126 strain, the genes corresponding to Ade B pump (AU097_02660), membrane protein (AU097_03195), class C 
extended-spectrum $\beta$-lactamase ADC-26 (AU097_00745) which upregulated with 3.2339, 2.3114 and $3.5588 \log _{2}$-fold change, respectively, in RNA-sequencing data, showed $3.9497,2.3170$ and $6.5163 \log _{2}{ }^{-}$ fold change, respectively, using the qRT-PCR analysis. The genes that downregulated in the RNAsequencing data, transfer RNA (AU097_11755), iron-containing alcohol dehydrogenase (AU097_18615) and aldehyde dehydrogenase (AU097_18630) with -1.0926, -4.0487 and $-3.0715 \log _{2}$-fold change, respectively, showed $-0.4585,-4.0264$ and $-2.464 \log _{2}$-fold change using the qRT-PCR analyses, respectively.

\section{Transmission electron micrograph of OMVs}

Transmission electron micrograph images from the negatively stained $A$. baumannii showed the presence of OMVs in both the ATCC 19606, JU0126 control and treated strains with an abundance of OMVs observed from the treated strains (Fig. 3A-D). It is known that OMVs are associated with bacterial survival, nutrient uptake, environmental stress and biofilms [18]; and, this is evident in the present study with an increased OMV presence in strains exposed to eravacycline induction.

\section{Effect of eravacycline induction on the OMV proteome}

The OMVs proteome of eravacycline treated and control ATCC 19606 and clinical strain JU0126 were analyzed using LC-MS/MS study which resulted in the identification of 227 and 342 proteins for control ATCC 19606 and treated, respectively. Similarly, 203 and 265 proteins were identified for the OMVs from JU0126 control and treated strains, respectively (Additional file 4). These proteins were analyzed further using pSORTb v3.0.2 and SignalP v5.0. The occurrence of Omp38 and entericidin EcnA/B family proteins were of high intensity within the OMVs taken from the eravacycline treated clinical strain JU0126 based on the LC-MS/MS proteome analysis. Apart from the above two highly enriched proteins, a total of 10 other Omp proteins were also identified in the OMVs from eravacycline induced clinical strain JU0126. Overall, the resistance-associated proteins in OMVs identified from the proteome analysis of the eravacycline treated clinical strain JU0126 were: porin, outer membrane porin D, ABC transporter, substrate-binding protein family $\mathrm{V}, \mathrm{OmpA}$ family protein, Omp38, Omp transport protein Ompp1, putative acriflavine resistance protein $\mathrm{A}$, transcriptional regulators $\mathrm{AraC}$ and TetR family, major facilitator family protein and $\beta$-lactamase. The same OMP repeated to have high intensity in the OMVs from ATCC 19606 strain, and they are Omp38 and entericidin EcnA/B family proteins. Resistance proteins present in the OMVs from eravacycline treated ATCC 19606 strain, includes porin, Ompp1, OmpA family protein, OmpW, Omp38, Omp85, OprM efflux pump, OprD, GntR regulator and TetR regulator.

\section{Subcellular localization of proteins from OMVs}

The subcellular localization of the 254 OMV proteins from the eravacycline-untreated control $A$. baumannii ATCC 19606 strain (Fig. 4A) showed that a major number of proteins were localized to cytoplasmic (116), while 40 came under cytoplasmic membrane, 29 outer membranes, 5 periplasmic, 3 extracellular and 61 unknowns. The subcellular localization of the $342 \mathrm{OMV}$ proteins from the eravacycline treated A. baumannii ATCC 19606 strain showed that major number of proteins were again 
cytoplasmic (107), 74 cytoplasmic membranes, 11 outer membranes, 33 periplasmic, 20 extracellular and 97 unknown sites.

The comparison of the pie distribution of the protein localization among the antibiotic-induced and uninduced strains showed the difference in the total number of proteins. In the A. baumannii ATCC 19606 strain (treated), eravacycline induction (Fig. 4B) resulted in the increase in proteins pertaining to different functions: cytoplasmic membrane proteins with antibiotic resistance functionality included outer membrane family proteins, outer membrane assembly complexes, OMP, OMP assembly factor, putative RND efflux pumps, carbapenem-associated resistance proteins, and OXA-51 family carbapenemhydrolyzing class $D \beta$-lactamase OXA-98. Stress tolerance proteins, peptidases, transcription termination factors, and many ribosomal proteins were also localized in the cytoplasmic membrane. Antibiotic resistance-related proteins localized in the outer membrane, includes Metallo $\beta$-lactamases-fold metallohydrolase, OmpW family protein, outer membrane insertion signal domain protein, $A B C$ transporter family protein, ompA family protein and along with ribosomal proteins. Cytoplasmic proteins expressed were OMP transport protein, Ompp1/FadL/TodX family, outer membrane efflux protein OprM, ATP-binding cassette protein along with ribosomal proteins, elongation factors, and transcriptional regulator.

Proteins from the OMVs isolated from the untreated control of clinical isolate A. baumannii JU0126 (Fig. 4C) were screened for their cellular localization. Of the 214 proteins, 73 were cytoplasmic, 25 cytoplasmic membranes, 27 outer membranes, 16 periplasmic, 18 extracellular and 55 belonged to unknown sites. Of the 265 proteins from eravacycline treated $A$. baumannii JU0126 strain (Fig. 4D), 71 were cytoplasmic, 78 cytoplasmic membranes, 56 outer membrane, 13 periplasmic, 15 extracellular and 32 belonged to unknown sites.

\section{Functional annotation of proteins from OMVs}

The annotation of the differentially expressed protein was done using the STRAP tool that uses an exhaustive database of Uniprot, EBI and GO to classifies the proteins based on their biological process, cellular component and molecular function [19]. The proteins from the eravacycline treated A. baumannii ATCC 19606 and control strains were associated majorly with the molecular function term, binding activity (125 and 94 proteins) and catalytic activity (123 and 99 proteins), others were structural molecule (42 and 23 proteins) or involved with the role of antioxidant activity (4 and 1 protein), molecular transducer ( 1 and 1 protein) and many other roles (15 and 11 proteins), respectively (Fig. 4E). Proteins annotated in the OMVs from the clinical strain A. baumannii JU0126 treated and control were implicated with the binding activity ( 90 and 62 proteins) and catalytic activity (77 and 63 proteins), structural molecule (35 and 18 proteins), antioxidant activity ( 2 and 1 protein), molecular transducer ( 1 and 1 protein) and roles (12 and 13 proteins), respectively (Fig. 4F).

Presence of enriched genes and proteins functioning as virulence factors and resistance determinants 
The genes (functions pertaining to virulence, stress response and antibiotic resistance) expressed (from RNA sequencing) in the eravacycline induced A. baumannii strains of ATCC 19606 and JU0126 were compared with the uninduced controls with respect to their $\log _{2}$-fold change (Fig. 5A, B). A. baumannii has many innate virulence factors and resistance proteins, many of which have been described in detail by Lee et al. [20]. Protein secretion systems are among the major virulence factors in Gram-negative bacteria, they function by assisting in the process of transporting proteins between cellular locations [21]. Genes were considered as differentially expressed when the $\log _{2}$-fold change was $>2$-fold.

The mRNA expression data were compared with the protein abundance dataset based on their differential expression (Additional file 5). The correlation between mRNA expression and protein expression for all the genes from OMVs in both the treated and control strains of ATCC 19606 and JU0126 was represented with a correlation coefficient. Overall, comparing mRNA and protein expression from our data, there was a very low correlation ( $r=0.0184$ from ATCC 19606 and $r=0.0038$ for JU0126). Figure 6A represents the correlation between whole-gene mRNA expression and OMV proteome based on both $\log _{2}$-fold change and $P$-value for the strain A. baumannii ATCC 19606 and Figure 6B the same for JU0126 strain. In the strain A. baumannii JU0126, very few proteins displayed linear correlation with the similar expression pattern in whole-cell mRNA and OMV protein abundance; they are 30 S ribosomal proteins S9, S3, S5, 50S ribosomal proteins L2, L16, L1, L18 and L28.

\section{DEGs/proteins belonging to the most highly enriched biological pathways}

Two PPI networks were constructed using string database for ATCC 19606 and JU0126 strains. The commonly expressed gene/proteins from both transcriptome and OMVs proteome were selected and used to build the PPI network. For ATCC 19606, 328 nodes and 3603 edges and 83 nodes and 545 edges for JU0126 were generated from string database (Additional file 6). Both ATCC 19606 and JU0126 PPI networks were visualized using Cytoscape. The $P$-value of mRNA versus protein from both ATCC 19606 and JU0126 were used for node size and combine score for edge size generation in PPI network. Using ClueGO/CluePedia plug-in of Cytoscape software, enrichment pathways for commonly identified genes/proteins from both mRNA and OMVs proteome were analyzed. For A. baumannii 19606, high enrichment of biological processes belonging to "ribosome", "RNA polymerase", "regulation of translation", "nucleoside phosphate", "purine nucleotide metabolic process", "rRNA binding" and "tRNA binding" were found in the functional analysis. In the functional analysis, high enrichment of processes pertaining "ribosome"," ribosomal subunit" and "RNA polymerase" were identified in A. baumannii JU0126 (Fig. 7A, B).

Highly interactive and subgraph network was generated using the MCODE plug-in from Cytoscape software. For A. baumannii ATCC 19606, 14 efficient clusters and 4 for JU0126 strain were identified, for further analysis nodes with $n>10$ clusters were selected from both strains (Additional file 7). Four clusters for ATCC 19606 were selected, the first cluster consisted of 54 nodes with a score of 51.32, the second, third and fourth clusters had 11,18 and 11 nodes with scores 10.6, 9.8 and 7.2, respectively. Cluster one consisted majorly of ribosomal proteins, proteins for RNA polymerases, elongation factors, 
intracellular organelles, ribosomal subunits, tRNA binding and regulation of translation, cluster two included cell envelope organization and cluster three with response to toxic substance. For the strain JU0126, only one cluster was taken with 25 nodes and 23.3 scores, includes ribosomal subunit, RNA polymerase, cellular macromolecules biosynthesis, cellular nitrogen compound biosynthesis. The enlarged view of each cluster are represented in Additional file 8 2A-D for ATCC 19606 and Supplementary Figure 2E for JU0126 respectively.

\section{Discussion}

In the present study, A. baumannii ATCC 19606 and tigecycline resistant clinical isolate A. baumannii strain JU0126 were induced in vitro for resistance to eravacycline. Whole-cell transcriptome analysis was performed for both eravacycline induced and non-induced strains of A. baumannii strains. In addition, OMVs were isolated from both strains and their proteomes studied from both eravacycline induced ATCC 19606 and JU0126 strains. The whole-cell transcriptome expression was compared with OMVs proteome in both ATCC 19606 and JU0126 strains.

\section{Upregulated DEGs/proteins}

Genes pertaining to the family of drug efflux and membrane transport were significantly high in expression among both ATCC 19606 and JU0126 strains. The genes that were upregulated in the eravacycline treated ATCC 19606 strain in comparison with untreated strains included majorly of efflux and transporter families. The multidrug efflux RND transporter permease subunit gene and major facilitator superfamily (MFS) transporter were significantly overexpressed in the ATCC 19606 treated strain. Although specifically, AdeB pump and some membrane proteins were upregulated in the eravacycline treated JU0126 strain. The eravacycline-based antibiotic induction in bacterial strains leading to the upregulation of MDR pumps can be supported through some similar prior works. Abdallah et al. (2015), in their study, showed that the increased MIC values to eravacycline up to $4 \mu \mathrm{g} / \mathrm{mL}$ corresponded to increase in the expression of AdeABC MDR pump. However, this upregulation does not always signify the resistance towards the induced antibiotic. As is the case, that no MDR specific resistance towards eravacycline has been reported in Acinetobacter [22]. The enzyme M1 family peptidase is present in many pathogens and is known to be a key enzyme for the survival in these organisms. It was notable that these enzymes were also upregulated in the antibiotic-treated ATCC 19606 strain, signifying the pressure of survival as induced by the presence of antibiotic. RND efflux pumps are a common mechanism involved in antibiotic resistance among $A$. baumannii, AdeB efflux pump is one of the upregulated proteins in the antibiotic-induced JU0126 strain. In A. baumannii, AdeABC is the first characterized efflux pump belonging to the RND superfamily. The operon codes for a major facilitator superfamily protein transporter protein AdeA, a multidrug transporter AdeB, and an outer membrane protein (OMP) AdeC. Eravacycline resistance in A. baumannii due to AdeABC efflux has not been reported before; hence, this upregulation can be attributed to the induction due to the antibiotic even though it is not a substrate for the pump. Antibiotic induced $a d e B$ efflux pump resistance has a major influence on the resistance status of $A$. baumannii [23]. The role of similar overexpression of adeB has been noted in 
some MDR isolates resistant to tigecycline in some of the previous research works [24]. Eravacycline induced overexpression of macA efflux protein of the MacAB-TolC MDR pump expression was reported in K. pneumoniae emphasizing the role of efflux in eravacycline heteroresistance [25]. The next protein that was upregulated in the present study was an Omp38, which is a major porin protein from $A$. baumannii. OMPs are crucial proteins for antibiotic diffusion and membrane permeability; deficiency of which leads to increased susceptibility to antibiotic. Studies have shown increased production of OMPs, like OmpA38, CarO, OmpW, in the presence of tetracycline, suggesting that the overexpression relates to overcoming antibiotic stress [26]. A. baumannii is an organism that harbors multiple mechanisms for antibiotic resistance, and $\beta$-lactamases are a group that tackles the $\beta$-lactam drugs efficiently in these organisms. Class $C$ extended-spectrum $\beta$-lactamase $A D C-26$ was seen upregulated in our study in JU0126 strain. The overexpression of ADC is reported to confer resistance to a range of $\beta$-lactam antibiotics making the infections caused by $A$. baumannii difficult to treat [27]. However, the overexpression in the eravacycline treated JU0126 could be due to a random antibiotic stress response because these $\beta$-lactamases does not have substrate specificity for a non- $\beta$-lactam drug.

Previous reports have demonstrated that OMVs isolated from antibiotics resistance strains help susceptible strains in transferring antibiotics resistance genes and proteins under antibiotic stress condition [18]. Carbapenem-resistant $A$. baumannii releases OMVs packed with carbapenem resistancerelated genes and could undertake the horizontal transfer to carbapenem-susceptible A. baumannii [28]. In one study, OMVs from E. coli were found to seize antibiotics, such as colistin and degrade the antimicrobial peptides like melittin [29]. Moraxella catarrhalis and Staphylococcus aureus also releases OMVs, which carries $\beta$-lactamase helping the bacteria to survive in the presence of $\beta$-lactam antibiotics [30].

\section{Downregulated DEGs/proteins}

The tetracycline group of antibiotics act by binding to ribosomal subunit 30S thereby blocking the aminoacyl-tRNA to bind to ribosomal acceptor site A; hence, inhibiting the protein synthesis [31]. It was reported by Vrentas et al., that the downregulation of RNA synthesis occurs as a result of protein synthesis inhibition [32]. In the present study, the transfer RNAs were downregulated in both the ATCC 19606 and JU0126 strain which explains the adaptation of the bacterium to pressure, trying to keep the metabolic process minimal, similar reports on the reduced metabolism due to tigecycline induction was done by Liu et al.[33].

In our study, porin proteins were downregulated in the A. baumannii ATCC 19606 strain. The loss or downregulation of porins is a mechanism of resistance, wherein the bacteria reduce the cell permeability preventing antibiotic entry and decrease the susceptibility [34]. The presence of tetracycline leads to differential expression of porins proteins, either increase or decrease of which decides the permeability of the cell envelope. The downregulation of porins in A. baumannii in this present study corresponds to the previous claims on tetracycline leading to the downregulation of numerous porins in Escherichia coli strains [35]. 


\section{Subcellular localization of proteins from OMVs}

The subcellular localization of the proteins expressed in the OMVs from both the eravacycline treated and untreated control strains was identified using pSORT-B 3.0. Their results give crucial information on the function of the protein, which can be compared with their expression pattern in the present study condition (upregulated or downregulated; antibiotic stress or antibiotic resistance). The pSORT-B categorizes the Gram-negative bacterial proteins into five major sites-the cytoplasm, the inner membrane, the periplasm, the outer membrane and the extracellular space [36].

The localization analysis in the current study was done to visualize the effect of antibiotic stress on the OMVs specifically focusing on their proteins and its functions. In both the ATCC19606 and JU0126 strains, proteins with functions related to resistance and stress were predominant, like the outer membrane proteins, efflux pumps, $\beta$-lactamase associated resistance proteins, stress tolerance proteins and peptidases. It is known that the proteins from OMVs aid the invasiveness of the bacteria, and are enriched with toxins, bioactive and virulence proteins. OMVs are a key for bacterial survival with their role in bacterial self-defense, formation of biofilm, antibiotic resistance and host-immune response modulation [37], [38]. The exposure of cells to environmental contaminants (antibiotics) has potentially evolved bacterial OMVs, either with multidrug efflux pumps capabilities or with ability to catalyse degradation by sequestering antibiotics from the extracellular milieu [39], [40].

\section{DEGs pertaining to virulence factors}

The proteins belonging to the type VI secretion systems (T6SSs), which are a new type among the bacterial secretion systems, were increased in their expression ranging from 18-fold to a minimum of 7fold change in the A. baumannii JU0126 eravacycline treated strain compared with the control. T6SSs are associated with the pathogenicity in bacteria with the experimentally proven role in bacterial virulence [41]. T6SSs does a bacteriophage-like contractile injection of effector proteins puncturing into target cells and when they inject antibacterial toxins to competing for bacterial cells, they become 'antibacterial' T6SSs [42], [43]. Some of the other genes with differential expression in JU0126 strain alone were LuxR family transcriptional regulator (a crucial protein involved with quorum sensing) with a 7-fold change in expression. These proteins coordinate the expression of virulence factors, biosynthesis of antibiotics and transfer of plasmids, bioluminescence, and formation of biofilms [44].

The efficient induction of eravacycline resistance was evident with the 5 -fold change in expression of the gene that encodes $50 \mathrm{~S}$ ribosomal protein L2, which is an rRNA binding protein and helps in the interaction of $30 \mathrm{~S}$ and $50 \mathrm{~S}$ subunits in order for tRNA binding to happen and; hence, peptide bond formation [45]. This contradicts the action of eravacycline which negates the bacterial protein synthesis by binding to the $30 \mathrm{~S}$ ribosomal subunit, stopping peptide chains formation [9]. In addition, along with ribosomal proteins, genes for the elongation factor $\mathrm{G}$ were increased in their expression by 4 -fold change, EF-G has two roles; one, during the translocation and the other, in the ribosome disassembly [46]. Genes 
coding for the protein involved with cell metabolism, such as the d-alanyl-d-alanine carboxypeptidase, a serine peptidase was 4 -fold differentially expressed. These proteins are associated with virulence in Acinetobactersp. [47] and have been experimentally proved to be essential for intracellular replication in some bacteria [48].

The arginine succinyltransferase $\mathrm{A}$ (ast $\mathrm{A}$ ) enzyme [49] gene had an 8-fold increase in the expression of $A$. baumannii ATCC 19606 treated strain. AstA was found associated with healthcare-associated pathogen A. baumannii strains [50], and it has been attributed to the pathogenesis in other bacterial strains like uropathogenic E. coli (UPEC), and was reported as one of the virulence proteins in E. coli [51].

In both A. baumannii ATCC 19606 and JU0126 strains induced by eravacycline, the genes for type I secretion system and elongation factor TU had a positive $\log _{2}$-fold change, with a 3 -fold change in the JU0126 strain. The type I secretion system helps in the secretion of proteins from cytoplasm to the extracellular region. They harbor a specific OMP for their export and one among the best-studied is TolC from E. coli [52]. Elongation factor TU is a GTPase also known to perform moonlighting functions on the surface of human pathogens acting as a multifunctional adhesin [53].

\section{DEGs as resistance determinants}

Positive differential expression of many genes encoding resistance proteins was observed in both ATCC 19606 and JU0126 strains induced with eravacycline from the RNA sequence analysis. The efflux pumps and the ribosomal protection are the two main resistance mechanisms in A. baumannii to tetracycline class of drugs. In A. baumannii ATCC 19606, genes for all the major efflux pump family proteins had a positive differential expression, such as MFS, RND, multidrug and toxic compound extrusion (MATE) and ABC transporters. A 9-fold change in the expression of gene that codes for MFS transporter, many of which are involved in the drug efflux of antimicrobials, such as tetracyclines, fosfomycin, colistin and erythromycin [54] noted in the ATCC 19606 strain, whereas the JU0126 strain had a negative $\log _{2}$ change in the expression of this transporter. Tet efflux pumps are among the main types that come under MFS transporters, tet $A$ gene codes for an efflux protein that confers resistance to tetracyclines. The $A$. baumannii has two pump proteins under MFS category (uses proton exchange for a tetracycline-cation), Tet(A) and Tet(B) [55].

A 7-fold increase in the expression of TetR/AcrR family transcriptional regulator gene was observed in the A. baumannii ATCC 19606, induced with eravacycline while their expression in JU0126 strain showed a negative $\log _{2}$-fold. The TetR family of regulators (TFR) comes under the signal transduction systems with the drug-efflux pump regulation as their functional role. The expression of acrAB efflux pump operon is repressed by the AcrR. TetR is a family of tetracycline transcriptional regulator that has a role in the transcriptional control. In the absence of tetracycline antibiotic, TetR binds to the Tet(A) gene to repress its expression. Tet(A) exports tetracycline from the cell before it can exert the protein synthesis inhibition [56]. 
The overproduction of RND pumps, such as AdeABC, AdeFGH, and AdelJK is a major factor contributing to the resistance in Acinetobacter[57]. The gene for AdeB/AdeJ proteins had 3-fold differential expression in both the ATCC 19606 and JU0126 strains. AdeB is the multidrug transporter for the AdeABC tripartite efflux pump that expels out an array of antibiotics, such as aminoglycosides, $\beta$-lactams, chloramphenicol, erythromycin, and tetracyclines. This positive differential expression of AdeB can be correlated with the prior studies on which it was reported to be the most prevalent with increased expression among the MDR A. baumannii strains in Zhenjiang, China by Yang et al. [58]. Positive 6-fold differential expression of the multidrug efflux RND transporter permease subunit gene was noted in the ATCC 19606 strain, whereas a negative 3-fold decrease in the expression of the JU0126 strain. The ABC transporter ATP-binding protein gene expression was increased by 6-fold in the eravacycline induced ATCC19606 strain when compared with the uninduced strain; however, the MacB protein subunit was underexpressed with a negative 2-fold change in the same strain. The MacA-MacB-TolC is a three protein efflux system that expels out mainly macrolide class of antibiotics, and their expression may not be influenced in a large way by the eravacycline [59]. MATE family pumps are not much related to resistance towards the tetracycline class of drugs and basically confer resistance towards fluoroquinolones and imipenems [60]. However, there was a 7-fold change in the gene expression of MATE family pumps in the eravacycline induced $A$. baumannii ATCC 19606, but negative differential expression of negative 5-fold change in the JU0126 strain. Porins are the channel-forming protein that helps in the transport of molecules across the selectively permeable bacterial membrane bilayer. Mutations or changes in the porin proteins, such as loss or modification of the size of porin or lower expression result in the limited diffusion of $\beta$-lactams, fluoroquinolones, tetracycline and chloramphenicol [61]). Many of the genes coding porins had both positive and negative-fold change and reduced differential expression among both the A. baumannii ATCC 19606 and JU0126 strains treated with eravacycline like the carbapenem susceptibility porin CarO (-4- and -0.2-fold change), OmpW family protein (-3- and -8-fold change), outer membrane porin OprD family (5- and -4-fold change) and OmpA family protein ( -0.06 and 3 -fold change). This reduction in the expression of these porins signifies their role in conferring resistance by decreasing the antibiotic entry into cell.

Although $\beta$-lactamase enzyme production is not related to the eravacycline resistance, few classes of $\beta$ lactamase were noted to have both positive and negative-fold change. The genes for enzymes MBL-fold metallohydrolase had 5-fold change and 4-fold change, OXA-51 family carbapenem-hydrolyzing class-D $\beta$-lactamase OXA-259 with 1 and -0.4-fold change and class $C$ extended-spectrum $\beta$-lactamase ADC-26 with -1- and 7-fold change for A. baumannii ATCC 19606- and JU0126treated strains, respectively.

\section{OMVs proteins with function pertaining to stress and resistance}

The proteins involved with virulence, stress response and antibiotic resistance expressed in the OMVs of eravacycline induced A. baumannii strains of ATCC 19606 and JU0126 were compared with the uninduced controls with respect to their $\log _{2}$-fold change (only proteins with more than $2 \log _{2}$-fold change 
are mentioned below). Many proteins especially ribosomal proteins had more than $2 \log _{2}$-fold change in the expression in both the ATCC 19606 and clinical strain JU0126 and apart from that chaperons, OMP and resistance-conferring proteins were observed. Prior studies have also reported many OMP [62], [63] and resistance-conferring proteins expressed in OMVs of antibiotic-treated strains, our study identified many OMP and antibiotic resistance-related proteins from both A. baumannii ATCC 19606 and JU0126. In the ATCC 19606 strain, highest $\log _{2}$-fold change was for OmpA family protein (5.66), followed by Omp38 (4.43), $\beta$-lactamase (3.40), OprD family (2.91) and putative acriflavine resistance protein A (2.30). Other proteins pertaining to virulence, stress and bacterial survival with more than $2 \log _{2}$-fold change were copper-exporting ATPase (9.65) which is a copper tolerance protein, toluene tolerance protein Ttg2D (8.87), TonB-dependent siderophore receptor (6.69), 50S ribosomal proteins L14, L6, L4, L19, L16, L29 and L2 ( $\log _{2}$-fold change range from 2 to 6 ), 30S ribosomal proteins S11, S3 and S7 ( $\log _{2}$-fold change $3-$ 4), peptidases $S 41$ family (6.53), peptidoglycan-associated protein (6.0), type IV pilus biogenesis/stability protein PilW (4.94), type VI secretion protein, EvpB/VC_A0108 family (3.15), translation initiation factor IF3 (4.12), TolB belonging to the Tol-Pal peptidoglycan-associated lipoprotein system protein (3.34), chaperone protein HscA homolog that belongs to the heat shock protein 70 family (2.75) and vacJ-like lipoprotein. There were just two proteins associated with resistance showing more than $2 \log _{2}$-fold change in the OMV proteome of $A$ baumannii JU0126 strain, $\beta$-lactamase protein, and major facilitator family transporter. However, many stress response proteins, virulence, and survival proteins were expressed with more than $2 \log _{2}$-fold change in JU0126. The same as A. baumannii ATCC 19606, ribosomal protein abundance was very significantly high noting that the strains were induced resistance to eravacycline.30S ribosomal proteins S5, S4, S2, S3, S9 ranged from $2 \log _{2}$-fold change to $9 \log _{2}$-fold change and the $50 S$ ribosomal proteins $L 4, L 6, L 2, L 1, L 18, L 16, L 28, L 10$ with $\log _{2}$-fold ranging between 4 and 8.Other proteins like toluene tolerance protein Ttg2D, Tol-Pal system protein TolB, gammaglutamyltransferase, acetyl-CoA C-acetyltransferase, transcription termination factor Rho, YqaJ viral recombinase family protein, signal recognition particle protein, TonB-dependent siderophore receptor, and peptidases M48, S41 were expressed with more than $2 \log _{2}$-fold change in the eravacycline induced strains.

\section{Inconsistency in the expression patterns of OMVs Proteins in comparison to the bacterial whole gene expression profiles}

The overall results from the comparison of the two expression profiles, the protein, and the RNA were with a very low correlation coefficient. Some of the ribosomal proteins were upregulated in both RNA and OMV proteome expression profiles. The expression of ribosomal proteins in the OMV proteome can be supported by reports on the presence of RNAs and the proteins in involved in their synthesis. Sjöström et al. (2015) reported for the first time that RNAs were involved with bacterial OMVs [64]. Other proteins with a correlation between mRNA and protein expression include dihydrolipoamide acetyltransferase, DUF4142 domain-containing protein, class $C$ extended-spectrum $\beta$-lactamase ADC-26 and a hypothetical protein. In the strain A. baumannii ATCC 19606, although many ribosomal proteins showed upregulation in their expression, linear correlation of both mRNA and protein expression was seen only in proteins, 
copper-translocating P-type ATPase, methylmalonate-semialdehyde dehydrogenase (CoA acylating), adenosine deaminase and gamma-glutamyltransferase family g-protein. The low correlation of the mRNA and protein components based on the $\log _{2}$-fold change comparison suggests that proteins in OMVs are selectively enriched, transported from the bacterial cell and/or due to wide range of regulatory mechanisms involved in the post-transcriptional level [65]. A poor correlation of similar comparison was reported by Yun et al. (2018) in their study of proteins in OMVs and protein fractions from bacterial cell membranes. They have mentioned the reason to be that proteins in the OMVs are differentially selected and sorted from the host bacteria.

\section{Enriched biological pathways}

PPI networks from commonly expressed gene/proteins from both transcriptome and OMVs proteome of ATCC 19606 and JU0126 strains were constructed. Pathways that were found enriched were significantly ironically related to transcription and RNA synthesis, owing to the fact that the bacterium was grown in an eravacycline stressed environment and the subsequent induced resistance.

\section{Conclusions}

The transcriptome of the whole cell and OMVs proteome abundance was studied for two A. baumannii strains, one an ATCC 19606 and a clinical isolate JU0126 in an eravacycline induced antibiotic resistant condition. From the whole-cell RNA sequence analysis, different virulence factors, resistance genes were upregulated, whereas the OMVs proteome was enriched with more proteins essential for bacterial stress and survival. The network interactions and respective MCODE cluster information clearly correlate with the study growth conditions with high eravacycline concentrations and the induced resistance towards the antibiotic in the bacterium. The observation from this study is that eravacycline greatly upregulates the resistance-conferring genes in the whole cell, whereas not many resistance-related effects were seen in the OMVs proteome. This work focused on the differential proteome of OMVs and their possible influence in the induced resistance to eravacycline; however, it was found from the outcome of the results that OMVs rather support the bacterial survival with its stress proteins, chaperones and proteases more than the resistance-conferring abilities. OMVs are essential although not alone, but in close unison with the bacterial cellular factors for the resistance and sustenance in the lethal eravacycline concentrations.

\section{Methods}

\section{Bacterial strains}

MDR Acinetobacter baumannii clinical strain (JU0126) was collected from Jiangbin Hospital, Zhenjiang, Jiangsu Province, China, and also A. baumannii ATCC 19606 was used as a reference strain. Further, the minimal inhibitory concentration of eravacycline antibiotic was ascertained for both the strains.

\section{Induction of eravacycline resistance}


A single colony of both ATCC 19606 and JU0126 strain were inoculated into the cation adjusted MuellerHinton broth (CAMHB) containing sub-MIC concentration of eravacycline incubated at $37^{\circ} \mathrm{C}$ at $250 \mathrm{rpm}$ overnight. On day $3,0.1 \mathrm{~mL}$ culture suspension was transferred into the freshly prepared CAMHB $(10 \mathrm{~mL})$ with next higher concentration of eravacycline and incubated at $37^{\circ} \mathrm{C}$ at $250 \mathrm{rpm}$ overnight. This passage was continued until the maximum concentration above the MIC of eravacycline was achieved, that the strains were able to resist and grow in the same incubation conditions [66], [67].

\section{RNA sequencing}

The quality and quantity of the total RNA from both A. baumannii ATCC 19606 and JU0126 strains were assessed using the NanoPhotometer ${ }^{\circledR}$ spectrophotometer (IMPLEN, CA, United States) and Qubit ${ }^{\circledR}$ RNA Assay Kit in Qubit $\circledast 2$ 2.0 Flurometer (Life Technologies, CA, United States), respectively followed by RNA sequence analysis. The RNA integrity number (RIN) was calculated using the RNA Nano 6000 Assay Kit of the Bioanalyzer 2100 System (Agilent Technologies, CA, United States). The RNA-sequencing library was constructed using NEBNext ${ }^{\circledR}$ Ultra ${ }^{\text {TM }}$ Directional RNA Library Prep Kit for Illumina ${ }^{\circledR}$ (NEB, United States) as per the manufacturer's instructions.

The purification of library fragments was done using AMPure XP system (Beckman-Coulter, Beverly, United States), and $3 \mu \mathrm{L}$ USER Enzyme (NEB, United States) was used with size-selected, adaptor-ligated cDNA to get 150-200 bp sized cDNA. Phusion high-fidelity DNA polymerase, Universal PCR primers, and Index $(X)$ primers were used for the PCR; and AMPure XP system was used to purify the PCR products, and the quality was thus assessed using Agilent Bioanalyzer 2100 system. Clusters were generated using a cBot Cluster Generation System using TruSeq PE Cluster Kit v3-cBot-HS (Illumia), and the library preparations were sequenced using Illumina Hiseq platform.

\section{Analysis of the RNA-sequence data}

The sequenced libraries were mapped against predicted transcripts from the A. baumannii ATCC 19606 genome using TopHat v2.0.4. HTSeq v0.6.1 was used to count the read numbers mapped to each gene, an abundance of transcript (FPKM, fragments per kilobase of exon per million fragments mapped) and significant changes in transcript expression were estimated using Cufflinks v2.0.2. The read counts for the sequenced libraries were adjusted using edgeR program package through one scaling normalized factor, and this was followed by differential expression analysis of two conditions/groups (two biological replicates per condition) using the DESeq $\mathrm{R}$ package (1.18.0). GO seq R package was used for Gene Ontology (GO) enrichment analysis of differentially expressed genes (DEGs), and the statistical enrichment was done using STRAP software [19].

\section{Reverse transcriptase-quantitative PCR}

Gene expression was analyzed using a previously described method [68]. Briefly, total RNA was isolated from $1 \times 10^{9} \mathrm{~A}$. baumannii cells. After the treatment with DNase, RNA samples were taken for CDNA synthesis. The template cDNA was diluted to 1:100, and $2.5 \mu \mathrm{L}$ of which was added to SYBR green PCR 
master mix for each reaction and Applied Biosystems ${ }^{\text {TM }}$ StepOne ${ }^{\text {TM }}$ Real-Time PCR was used for the analysis. Both internal forward and reverse primers were designed using IDA website (Additional file 1). The experiments were repeated in independent triplicates. Normalization to the gyrB genes facilitated the calculation of the fold changes using the threshold cycle (Ct) method.

\section{Purification of OMVs}

OMVs of both A. baumannii ATCC 19606 and JU0126 were prepared from previously described methods [69]. In brief, both strains are grown in $500 \mathrm{~mL}$ of Luria Bertani (LB) broth until the OD at $600 \mathrm{~nm}$ reached 1.0 at $37^{\circ} \mathrm{C}$ in incubator shaker, then centrifuged at $6000 \mathrm{~g}$ at $4^{\circ} \mathrm{C}$ to remove bacterial cells. The supernatants were filtered through vacuum filter $(0.2 \mu \mathrm{m}$ size $)$ and OMVs were concentrated using Merck ultrafiltration tube. Then, the samples were taken for ultracentrifugation at $150,000 \mathrm{~g}$ at $4^{\circ} \mathrm{C}$ for $3 \mathrm{~h}$, pellets were resuspended in phosphate buffer saline and protein concentration was determined using modified BCA assay (Thermo Scientific). The OMVs were initially fixated, and the ultrathin sections were stained using 3\% uranyl acetate negative staining technique and imaged using Transmission Electron Microscope (Philips). Purified OMVs were checked for the sterility and stored at $-80^{\circ} \mathrm{C}$ for further use.

\section{LC-MS/MS analysis of OMVs}

OMVs proteins were identified by one-dimensional electrophoresis-liquid chromatography-tandem mass spectrometry using nano-LC LTQ-Orbitrap Mass Spectrometer, Thermo Fisher Scientific, Bremen, Germany. OMVs protein was trypsin digested, and each fraction was reconstituted in HPLC grade $5 \%$ acetonitrile and $0.1 \%$ formic acid (solvent $\mathrm{A}$ ) and then loaded on to the nano HPLC column. A gradient was formed, and the peptides were eluted with increasing concentration of $98 \%$ acetonitrile and $1 \%$ formic acid (solvent B). The eluted peptides were detected in the ESI mass spectrometer and produced a tandem mass spectrum of specific fragment ions for each peptide [70].

\section{Identification and quantification of proteins from OMVs}

LC-MS/MS raw data were used to identify the peptides/proteins from OMVs using MaxQuant (version 1.6.3.4) with match between runs, matching time window of $2 \mathrm{~min}$. The search parameters are as follows: enzymes specify-trypsin; variable modification-oxidation of methionine (15.995 Da); fixed modification-carbamidomethylation of cysteine (57.021 Da); two missed cleaves; precursor ions tolerance $-20 \mathrm{ppm}$ and fragment ions tolerance $-4.5 \mathrm{ppm}$. Reference proteome set of $A$. baumannii ATCC 19606 was retrieved from Uniprot database. Contaminant sequences were used for search and seven amino acids were set as the minimum length of peptide for analysis. The first majority proteins ID were selected and used for further analysis. Uniprot database and primary location were used to generate the protein location. Using DAVID web tool (https://david.ncifcrf.gov/), biological terms were generated and proteins identified from MS analysis were annotated for subcellular localization using pSORTb version 3.0.2 [36].

\section{Protein-protein interaction network (PPI) analysis for the gene/protein}


PPIs for A. baumannii ATCC 19606 and JU0126 strains were obtained from string database [71].

Potential PPIs were constructed for the common gene/protein from transcriptome and proteome analysis, respectively, using Cytoscape v3.7.1. The molecular complex detection (MCODE) algorithm was used to find highly interconnected subgraphs to find densely connected regions in the PPI network [72]. Using MCODE plug-in highly interconnected nodes $(n>10)$ were identified and clustered as subnetwork. Further, identified clusters from MCODE were used to find function enrichment using ClueGO/CluePediaplug-in of Cytoscape software [73], [74].

\section{Declarations}

\section{Ethics approval and consent to participate}

The clinical samples were taken as part of standard patient care and therefore no ethical approval was applied for their use. As this was an entirely in-vitro study using bacterial isolates ethical review is not required.

\section{Consent for publication}

Not applicable

\section{Availability of data and material}

Not applicable

\section{Competing interests}

The author(s) declare that they have no competing interests.

\section{Funding}

This work was supported by National Natural Science Foundation of China (Grant No. 81771756), a social development project of Jiangsu Province (Grant No. BE2016716), the Postdoctoral Foundation of Jiangsu Province (Grant No. 1601002C).

\section{Authors' contributions}


KD design of the study, performed expermients and analyses and helped to draft the manuscript, VA designed and helped drafted the manuscript. LW critically reviewed and edited the manuscript. JC and ZS helped in drawing figures and SW revised for its integrity and accuracy. $\mathbf{H X}$ approved the final version of this manuscript.

Kesavan Dinesh Kumar - KD, Vasudevan Aparna - VA囚Liang Wu - LW, Jianguo Chen - JC, Zhaoliang Su ZS, Shengjun Wang - SW, Huaxi Xu - HX.

\section{Competing interests}

The authors declare that they have no competing interests.

\section{Acknowledgements}

We thank to Jiangsu university for constant supporting this research study.

\section{Author Details}

${ }^{1}$ International Genomics Research Center (IGRC), Jiangsu University, Zhenjiang 212013, China

${ }^{2}$ Department of Immunology, School of Medicine, Jiangsu University, Zhenjiang 212013, China

${ }^{3}$ Department of Laboratory Medicine, The Affiliated People's Hospital, Jiangsu University, Zhenjiang 212001, China

\section{Abbreviations}

A. baumannii: Acinetobacter baumannii; OMVs: Outer membrane vesicles, MFS: major facilitator superfamily, resistance-nodulation-cell division superfamily: RND, Multi antimicrobial extrusion protein family: MATE, ATP-binding cassette superfamily: ABC, Outer membrane protein: OMP, American Type Culture Collection: ATCC, Jiangsu university strain No. 0126: JU0126, DEGs: differentially expressed genes, Multidrug resistance: MDR, ventilator-associated pneumonia: VAP, Liquid Chromatography with tandem mass spectrometry: $L C-M S / M S$, Cation adjusted Mueller-Hinton broth: CAMHB, Software Tool for Rapid Annotation of Proteins: STRAP, Luria Bertani: LB, Molecular Complex Detection: MCODE, Kyoto encyclopedia of genes and genomes: KEGG, Quantitative reverse transcriptase-polymerase chain reaction: qRT-PCR, Protein protein interaction network: PPI networks, Uropathogenic E. coli: UPEC, Arginine succinyltransferase $A$ : astA.

\section{References}


1. Garcia-Garmendia JL, Ortiz-Leyba C, Garnacho-Montero J, Jimenez-Jimenez FJ, Perez-Paredes C, Barrero-Almodovar AE, Gili-Miner M: Risk factors for Acinetobacter baumannii nosocomial bacteremia in critically ill patients: a cohort study. Clinical infectious diseases : an official publication of the Infectious Diseases Society of America 2001, 33(7):939-946.

2. Fournier $P E$, Richet $H$, Weinstein RA: The epidemiology and control of Acinetobacter baumannii in health care facilities. Clinical infectious diseases 2006, 42(5):692-699.

3. Lockhart SR, Abramson MA, Beekmann SE, Gallagher G, Riedel S, Diekema DJ, Quinn JP, Doern GV: Antimicrobial resistance among Gram-negative bacilli causing infections in intensive care unit patients in the United States between 1993 and 2004. Journal of clinical microbiology 2007, 45(10):3352-3359.

4. Bonomo RA, Szabo D: Mechanisms of multidrug resistance in Acinetobacter species and Pseudomonas aeruginosa. Clinical infectious diseases 2006, 43(Supplement_2):S49-S56.

5. Gales AC, Jones R, Sader HS: Global assessment of the antimicrobial activity of polymyxin B against 54731 clinical isolates of Gram-negative bacilli: report from the SENTRY antimicrobial surveillance programme (2001-2004). Clinical microbiology and infection 2006, 12(4):315-321.

6. Urban C, Mariano N, Rahal JJ, Tay E, Ponio C, Koprivnjak T, Weiss J: Polymyxin B-Resistant Acinetobacter baumanniiClinical Isolate Susceptible to Recombinant BPI21 and Cecropin P1. Antimicrobial agents and chemotherapy 2001, 45(3):994-995.

7. Kumarasamy KK, Toleman MA, Walsh TR, Bagaria J, Butt F, Balakrishnan R, Chaudhary U, Doumith $M$, Giske CG, Irfan $S$ et al: Emergence of a new antibiotic resistance mechanism in India, Pakistan, and the UK: a molecular, biological, and epidemiological study. The Lancet Infectious diseases 2010, 10(9):597-602.

8. Solomkin JS, Ramesh MK, Cesnauskas G, Novikovs N, Stefanova P, Sutcliffe JA, Walpole SM, Horn PT: Phase 2, randomized, double-blind study of the efficacy and safety of two dose regimens of eravacycline versus ertapenem for adult community-acquired complicated intra-abdominal infections. Antimicrob Agents Chemother 2014, 58(4):1847-1854.

9. Sutcliffe JA, O'Brien W, Fyfe C, Grossman TH: Antibacterial activity of eravacycline (TP-434), a novel fluorocycline, against hospital and community pathogens. Antimicrob Agents Chemother 2013, 57(11):5548-5558.

10. Solomkin J, Evans D, Slepavicius A, Lee P, Marsh A, Tsai L, Sutcliffe JA, Horn P: Assessing the Efficacy and Safety of Eravacycline vs Ertapenem in Complicated Intra-abdominal Infections in the Investigating Gram-Negative Infections Treated With Eravacycline (IGNITE 1) Trial: A Randomized Clinical Trial. JAMA surgery 2017, 152(3):224-232.

11. Abdallah M, Olafisoye $O$, Cortes C, Urban C, Landman D, Quale J: Activity of eravacycline against Enterobacteriaceae and Acinetobacter baumannii, including multidrug-resistant isolates, from New York City. Antimicrobial agents and chemotherapy 2015, 59(3):1802-1805.

12. Monogue ML, Thabit AK, Hamada Y, Nicolau DP: Antibacterial Efficacy of Eravacycline In Vivo against Gram-Positive and Gram-Negative Organisms. Antimicrobial agents and chemotherapy 2016, 
60(8):5001-5005.

13. Jones-Dias D, Carvalho AS, Moura IB, Manageiro V, Igrejas G, Canica M, Matthiesen R: Quantitative proteome analysis of an antibiotic resistant Escherichia coli exposed to tetracycline reveals multiple affected metabolic and peptidoglycan processes. Journal of proteomics 2017, 156:20-28.

14. Kulkarni HM, Jagannadham MV: Biogenesis and multifaceted roles of outer membrane vesicles from Gram-negative bacteria. Microbiology 2014, 160(10):2109-2121.

15. Yun SH, Park EC, Lee SY, Lee H, Choi CW, Yi YS, Ro HJ, Lee JC, Jun S, Kim HY et al: Antibiotic treatment modulates protein components of cytotoxic outer membrane vesicles of multidrugresistant clinical strain, Acinetobacter baumannii DU202. Clinical proteomics 2018, 15:28.

16. Young MD, Wakefield MJ, Smyth GK, Oshlack A: Gene ontology analysis for RNA-seq: accounting for selection bias. Genome biology 2010, 11(2):R14.

17. Kanehisa M, Goto S, Kawashima S, Okuno Y, Hattori M: The KEGG resource for deciphering the genome. Nucleic Acids Research 2004, 32(suppl_1):D277-D280.

18. Schwechheimer C, Kuehn MJ: Outer-membrane vesicles from Gram-negative bacteria: biogenesis and functions. Nature reviews Microbiology 2015, 13(10):605-619.

19. Bhatia VN, Perlman DH, Costello CE, McComb ME: Software tool for researching annotations of proteins: open-source protein annotation software with data visualization. Analytical chemistry 2009, 81(23):9819-9823.

20. Lee CR, Lee JH, Park M, Park KS, Bae IK, Kim YB, Cha CJ, Jeong BC, Lee SH: Biology of Acinetobacter baumannii: Pathogenesis, Antibiotic Resistance Mechanisms, and Prospective Treatment Options. Frontiers in cellular and infection microbiology 2017, 7:55.

21. Green ER, Mecsas J: Bacterial Secretion Systems: An Overview. Microbio/ Spectr 2016, 4(1).

22. Sutcliffe J, O'Brien W, Fyfe C, Grossman T: Antibacterial activity of eravacycline (TP-434), a novel fluorocycline, against hospital and community pathogens. Antimicrobial agents and chemotherapy 2013, 57(11):5548-5558.

23. Zhang T, Wang M, Xie Y, Li X, Dong Z, Liu Y, Wang L, Yang M, Song H, Cao H: Active efflux pump adeB is involved in multidrug resistance of Acinetobacter baumannii induced by antibacterial agents. Experimental and therapeutic medicine 2017, 13(4):1538-1546.

24. Coyne S, Courvalin P, Périchon B: Efflux-mediated antibiotic resistance in Acinetobacter spp. Antimicrobial agents and chemotherapy 2011, 55(3):947-953.

25. Zheng J-x, Lin Z-w, Sun X, Lin W-h, Chen Z, Wu Y, Qi G-b, Deng Q-w, Qu D, Yu Z-j: Overexpression of OqxAB and MacAB efflux pumps contributes to eravacycline resistance and heteroresistance in clinical isolates of Klebsiella pneumoniae. Emerging microbes \& infections 2018, 7(1):1-11.

26. Yun S-H, Choi C-W, Park S-H, Lee JC, Leem S-H, Choi J-S, Kim S, Kim SI: Proteomic analysis of outer membrane proteins from Acinetobacter baumannii DU202 in tetracycline stress condition. The Journal of Microbiology 2008, 46(6):720-727. 
27. Bhattacharya M, Toth M, Antunes NT, Smith CA, Vakulenko SB: Structure of the extended-spectrum class $\mathrm{C} \beta$-lactamase ADC-1 from Acinetobacter baumannii. Acta Crystallographica Section D: Biological Crystallography 2014, 70(3):760-771.

28. Rumbo C, Fernandez-Moreira E, Merino M, Poza M, Mendez JA, Soares NC, Mosquera A, Chaves F, Bou G: Horizontal transfer of the OXA-24 carbapenemase gene via outer membrane vesicles: a new mechanism of dissemination of carbapenem resistance genes in Acinetobacter baumannii. Antimicrob Agents Chemother 2011, 55(7):3084-3090.

29. Kulkarni HM, Nagaraj R, Jagannadham MV: Protective role of E. coli outer membrane vesicles against antibiotics. Microbiol Res 2015, 181:1-7.

30. Lee J, Lee EY, Kim SH, Kim DK, Park KS, Kim KP, Kim YK, Roh TY, Gho YS: Staphylococcus aureus extracellular vesicles carry biologically active beta-lactamase. Antimicrob Agents Chemother 2013, 57(6):2589-2595.

31. Roberts MC: Update on acquired tetracycline resistance genes. FEMS Microbiol Lett 2005, 245(2):195-203.

32. Ross W, Vrentas CE, Sanchez-Vazquez P, Gaal T, Gourse RL: The magic spot: a ppGpp binding site on E. coli RNA polymerase responsible for regulation of transcription initiation. Molecular cel/ 2013, 50(3):420-429.

33. Liu L, Cui Y, Zheng B, Jiang S, Yu W, Shen P, Ji J, Li L, Qin N, Xiao Y: Analysis of tigecycline resistance development in clinical Acinetobacter baumannii isolates through a combined genomic and transcriptomic approach. Sci Rep 2016, 6:26930.

34. Vaara M: Agents that increase the permeability of the outer membrane. Microbiology and Molecular Biology Reviews 1992, 56(3):395-411.

35. Lin X-M, Yang J-N, Peng X-X, Li H: A novel negative regulation mechanism of bacterial outer membrane proteins in response to antibiotic resistance. Journal of proteome research 2010, 9(11):5952-5959.

36. Yu NY, Wagner JR, Laird MR, Melli G, Rey S, Lo R, Dao P, Sahinalp SC, Ester M, Foster LJ: PSORTb 3.0: improved protein subcellular localization prediction with refined localization subcategories and predictive capabilities for all prokaryotes. Bioinformatics 2010, 26(13):1608-1615.

37. Jan AT: Outer membrane vesicles (OMVs) of Gram-negative bacteria: a perspective update. Frontiers in microbiology 2017, 8:1053.

38. Kim SW, Park SB, Im SP, Lee JS, Jung JW, Gong TW, Lazarte JMS, Kim J, Seo J-S, Kim J-H: Outer membrane vesicles from $\beta$-lactam-resistant Escherichia coli enable the survival of $\beta$-lactamsusceptible $E$. coli in the presence of $\beta$-lactam antibiotics. Scientific reports 2018, 8(1):5402.

39. Ciofu O, Beveridge TJ, Kadurugamuwa J, Walther-Rasmussen J, Høiby N: Chromosomal $\beta$-lactamase is packaged into membrane vesicles and secreted from Pseudomonas aeruginosa. Journal of Antimicrobial Chemotherapy 2000, 45(1):9-13.

40. Manning AJ, Kuehn MJ: Contribution of bacterial outer membrane vesicles to innate bacterial defense. BMC microbiology 2011, 11(1):258. 
41. Bingle LE, Bailey CM, Pallen MJ: Type VI secretion: a beginner's guide. Curr Opin Microbio/ 2008, 11(1):3-8.

42. Coulthurst SJ: The Type VI secretion system - a widespread and versatile cell targeting system. Res Microbio/ 2013, 164(6):640-654.

43. Kapitein N, Mogk A: Deadly syringes: type VI secretion system activities in pathogenicity and interbacterial competition. Curr Opin Microbiol 2013, 16(1):52-58.

44. Chen J, Xie J: Role and regulation of bacterial LuxR-like regulators. J Cell Biochem 2011, 112(10):2694-2702.

45. Cooperman BS, Wooten T, Romero DP, Traut RR: Histidine 229 in protein L2 is apparently essential for 50 Septidyl transferase activity. Biochemistry and cell biology = Biochimie et biologie cellulaire 1995, 73(11-12):1087-1094.

46. Wintermeyer W, Savelsbergh A, Konevega AL, Peske F, Katunin VI, Semenkov YP, Fischer N, Stark H, Rodnina MV: Functions of elongation factor $\mathbf{G}$ in translocation and ribosome recycling. In: Ribosomes. Springer; 2011: 329-338.

47. Yoon SH, Park YK, Kim JF: PAIDB v2.0: exploration and analysis of pathogenicity and resistance islands. Nucleic Acids Res 2015, 43(Database issue):D624-630.

48. Kikuchi H, Kim S, Watanabe K, Watarai M: Brucella abortusd-alanyl-D-alanine carboxypeptidase contributes to its intracellular replication and resistance against nitric oxide. FEMS Microbiol Lett 2006, 259(1):120-125.

49. Paixao AC, Ferreira AC, Fontes $M$, Themudo $P$, Albuquerque $T$, Soares $M C$, Fevereiro $M$, Martins $L$, Correa de Sa Ml: Detection of virulence-associated genes in pathogenic and commensal avian Escherichia coli isolates. Poultry science 2016, 95(7):1646-1652.

50. Wright MS, Haft DH, Harkins DM, Perez F, Hujer KM, Bajaksouzian S, Benard MF, Jacobs MR, Bonomo RA, Adams MD: New insights into dissemination and variation of the health care-associated pathogen Acinetobacter baumannii from genomic analysis. MBio 2014, 5(1):e00963-00913.

51. Luthje P, Brauner A: Virulence factors of uropathogenic E. coli and their interaction with the host. Advances in microbial physiology 2014, 65:337-372.

52. Delepelaire P: Type I secretion in gram-negative bacteria. Biochimica et Biophysica Acta (BBA)Molecular Cell Research 2004, 1694(1-3):149-161.

53. Widjaja M, Harvey KL, Hagemann L, Berry IJ, Jarocki VM, Raymond BBA, Tacchi JL, Gründel A, Steele JR, Padula MP: Elongation factor Tu is a multifunctional and processed moonlighting protein. Scientific reports 2017, 7(1):11227.

54. Pérez-Varela M, Corral J, Aranda J, Barbé J: Functional characterization of AbaQ, a novel efflux pump mediating quinolone resistance in Acinetobacter baumannii. Antimicrobial agents and chemotherapy 2018, 62(9):e00906-00918.

55. Vila J, Martí S, Sanchez-Céspedes J: Porins, efflux pumps and multidrug resistance in Acinetobacter baumannii. Journal of antimicrobial chemotherapy 2007, 59(6):1210-1215. 
56. Ramos JL, Martínez-Bueno M, Molina-Henares AJ, Terán W, Watanabe K, Zhang X, Gallegos MT, Brennan R, Tobes R: The TetR family of transcriptional repressors. Microbiol Mol Biol Rev 2005, 69(2):326-356.

57. Leus IV, Weeks JW, Bonifay V, Smith L, Richardson S, Zgurskaya HI: Substrate specificities and efflux efficiencies of RND efflux pumps of Acinetobacter baumannii. Journal of bacteriology 2018, 200(13):e00049-00018.

58. Yang H, Huang L, Barnie PA, Su Z, Mi Z, Chen J, Aparna V, Kumar D, Xu H: Characterization and distribution of drug resistance associated $\beta$-lactamase, membrane porin and efflux pump genes in MDR A. baumannii isolated from Zhenjiang, China. International journal of clinical and experimental medicine 2015, 8(9):15393.

59. Okada U, Yamashita E, Neuberger A, Morimoto M, van Veen HW, Murakami S: Crystal structure of tripartite-type ABC transporter MacB from Acinetobacter baumannii. Nature communications 2017, 8(1):1336.

60. Lin M-F, Lin Y-Y, Tu C-C, Lan C-Y: Distribution of different efflux pump genes in clinical isolates of multidrug-resistant Acinetobacter baumannii and their correlation with antimicrobial resistance. Journal of Microbiology, Immunology and Infection 2017, 50(2):224-231.

61. Fernández L, Hancock RE: Adaptive and mutational resistance: role of porins and efflux pumps in drug resistance. Clinical microbiology reviews 2012, 25(4):661-681.

62. McConnell MJ, Rumbo C, Bou G, Pachón J: Outer membrane vesicles as an acellular vaccine against Acinetobacter baumannii. Vaccine 2011, 29(34):5705-5710.

63. Kulkarni HM, Nagaraj R, Jagannadham MV: Protective role of E. coli outer membrane vesicles against antibiotics. Microbiological research 2015, 181:1-7.

64. Sjöström AE, Sandblad L, Uhlin BE, Wai SN: Membrane vesicle-mediated release of bacterial RNA. Scientific reports 2015, 5:15329.

65. Sun N, Pan C, Nickell S, Mann M, Baumeister W, Nagy I: Quantitative proteome and transcriptome analysis of the archaeon Thermoplasma acidophilum cultured under aerobic and anaerobic conditions. Journal of proteome research 2010, 9(9):4839-4850.

66. Lin M-F, Lin Y-Y, Lan C-Y: Induction of Tigecycline Resistance in Acinetobacter baumannii. BMC Microbiology 2014.

67. Boinett CJ, Cain AK, Hawkey J, Do Hoang NT, Khanh NNT, Thanh DP, Dordel J, Campbell JI, Lan NPH, Mayho M: Clinical and laboratory-induced colistin-resistance mechanisms in Acinetobacter baumannii. Microbial genomics 2019, 5(2).

68. Chang K-C, Kuo H-Y, Tang CY, Chang C-W, Lu C-W, Liu C-C, Lin H-R, Chen K-H, Liou M-L: Transcriptome profiling in imipenem-selected Acinetobacter baumannii. BMC genomics 2014, 15(1):815.

69. Jun SH, Lee JH, Kim BR, Kim SI, Park TI, Lee JC, Lee YC: Acinetobacter baumannii outer membrane vesicles elicit a potent innate immune response via membrane proteins. PloS one 2013, 8(8):e71751.

70. Kalli A, Hess S: Effect of mass spectrometric parameters on peptide and protein identification rates for shotgun proteomic experiments on an LTQ-orbitrap mass analyzer. Proteomics 2012, 12(1):21- 
31.

71. Szklarczyk D, Franceschini A, Kuhn M, Simonovic M, Roth A, Minguez P, Doerks T, Stark M, Muller J, Bork $\mathrm{P}$ et al: The STRING database in 2011: functional interaction networks of proteins, globally integrated and scored. Nucleic Acids Res 2011, 39(Database issue):D561-568.

72. O'Driscoll P, Merenyi E, Karmonik C, Grossman R: SOM and MCODE methods of defining functional clusters in MRI of the brain. Conference proceedings : Annual International Conference of the IEEE Engineering in Medicine and Biology Society IEEE Engineering in Medicine and Biology Society Annual Conference 2014, 2014:734-737.

73. Bindea G, Mlecnik B, Hackl H, Charoentong P, Tosolini M, Kirilovsky A, Fridman WH, Pages F, Trajanoski Z, Galon J: ClueGO: a Cytoscape plug-in to decipher functionally grouped gene ontology and pathway annotation networks. Bioinformatics 2009, 25(8):1091-1093.

74. Bindea G, Galon J, Mlecnik B: CluePedia Cytoscape plugin: pathway insights using integrated experimental and in silico data. Bioinformatics 2013, 29(5):661-663.

\section{Figures}



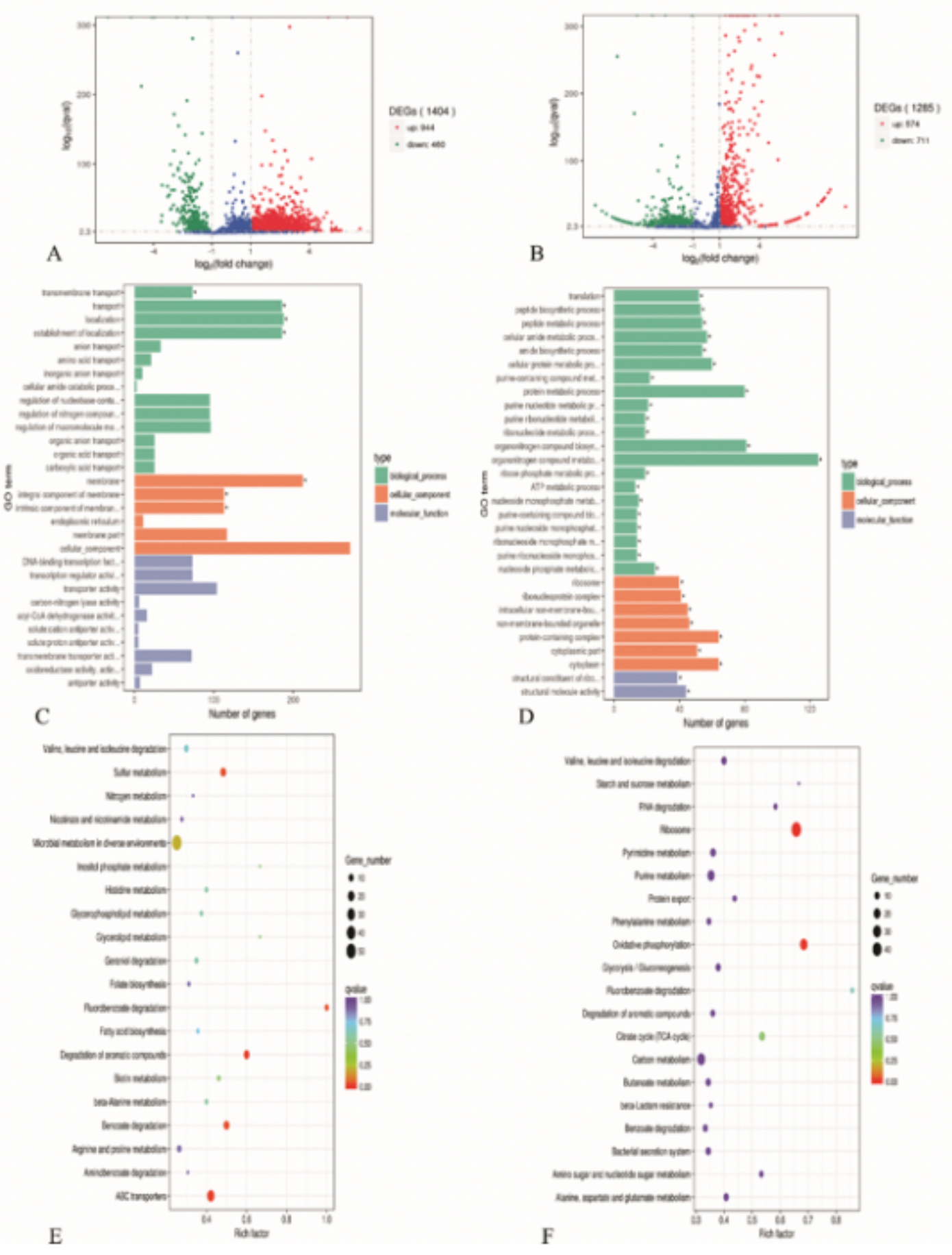

\section{Figure 1}

Comparison of differentially expressed genes (DEGs) between eravacycline treated and control samples of A. baumannii ATCC 19606 and clinical strain JU0126 


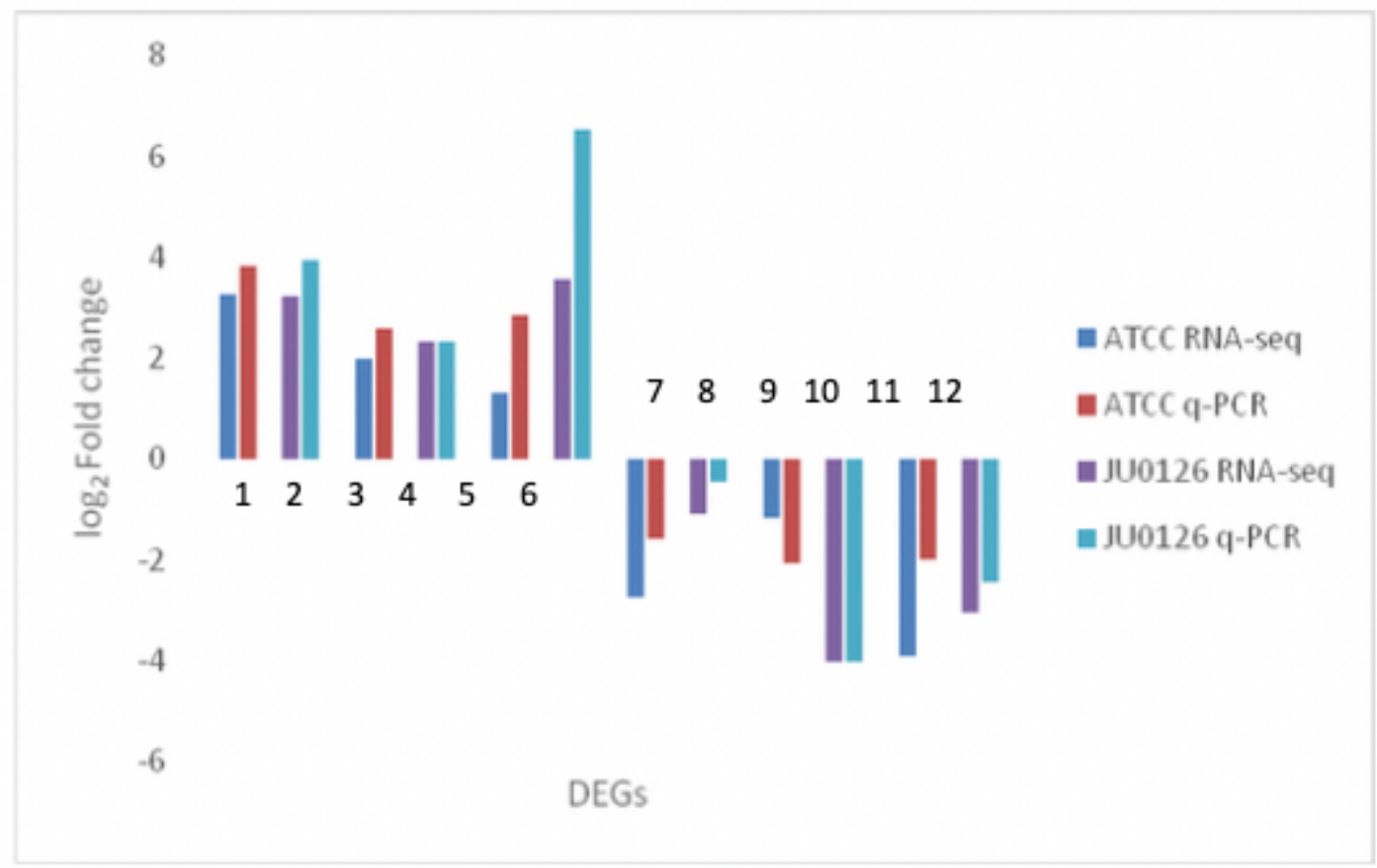

Figure 2

qRT-PCR analyses of six DEGs from each A. baumannii ATCC 19606 and JU0126 strain 


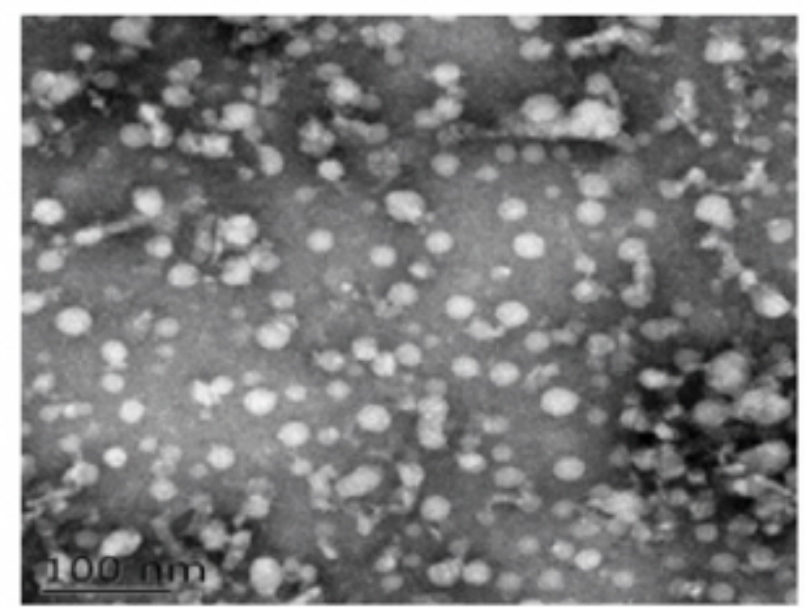

A. ATCC 19606 control

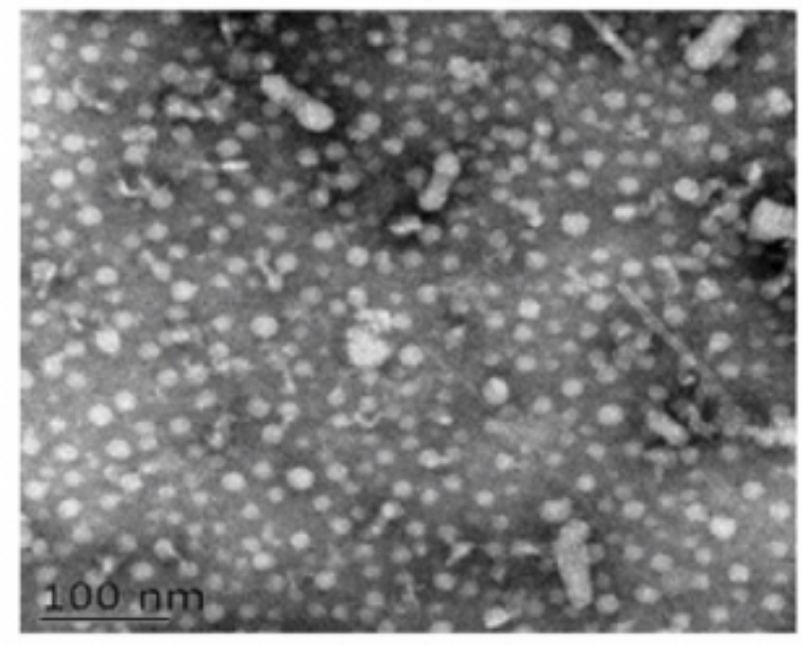

C. ATCC treated

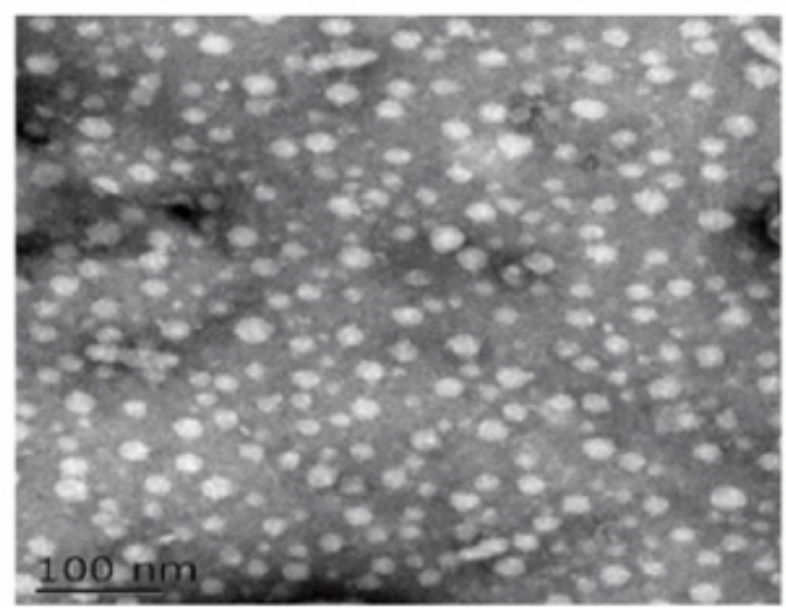

B. JU0126 control

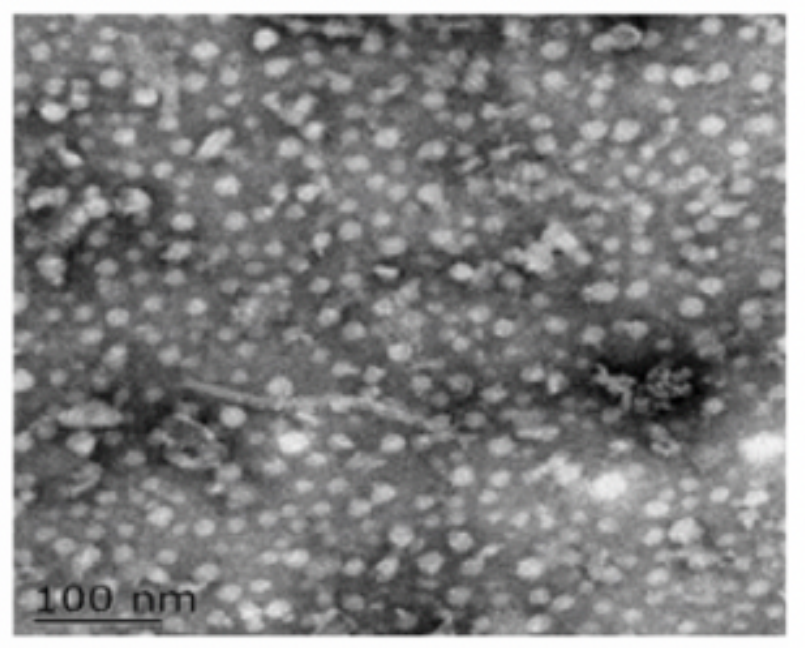

D. 126 treated

Figure 3

Transmission electron microscopic image of OMVs 

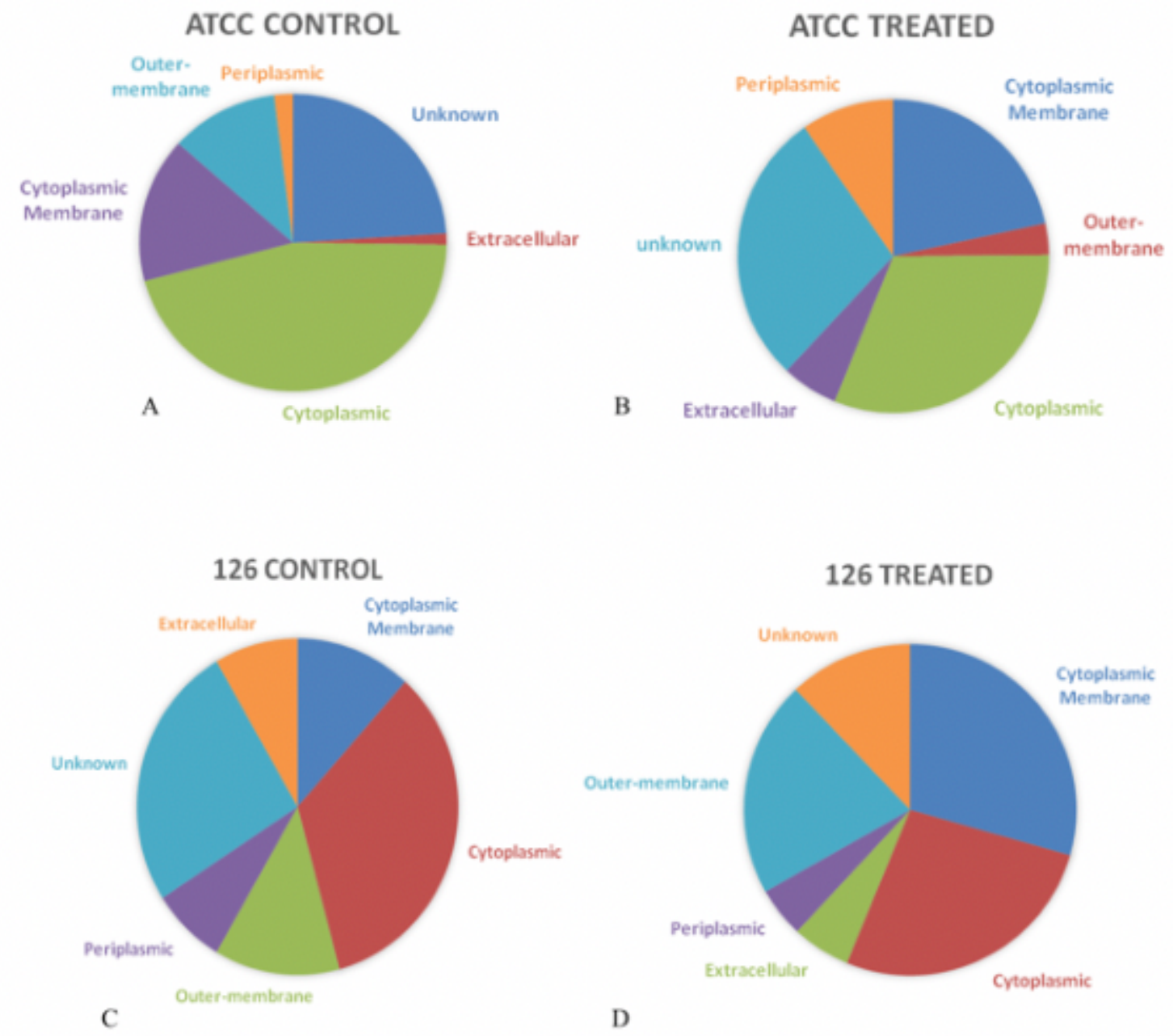

\section{TREATED}

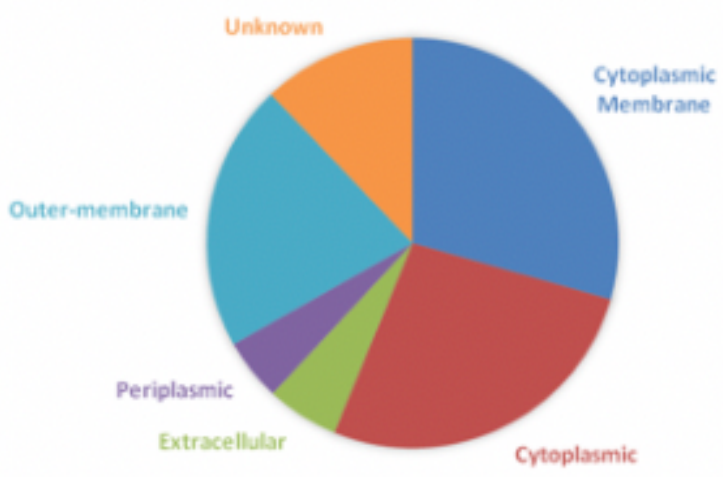

D
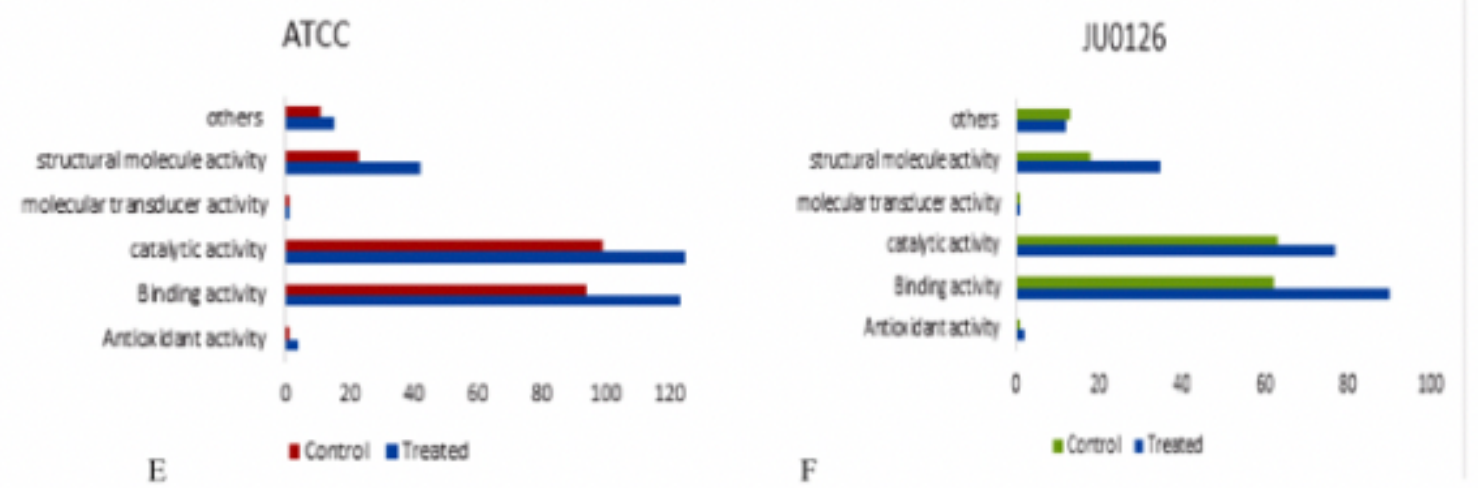

Figure 4

Classification of subcellular localization and Gene ontology annotations of OMVs proteins from control and eravacycline treated A. baumannii ATCC19606 and JU0126 strains using STRAP software 


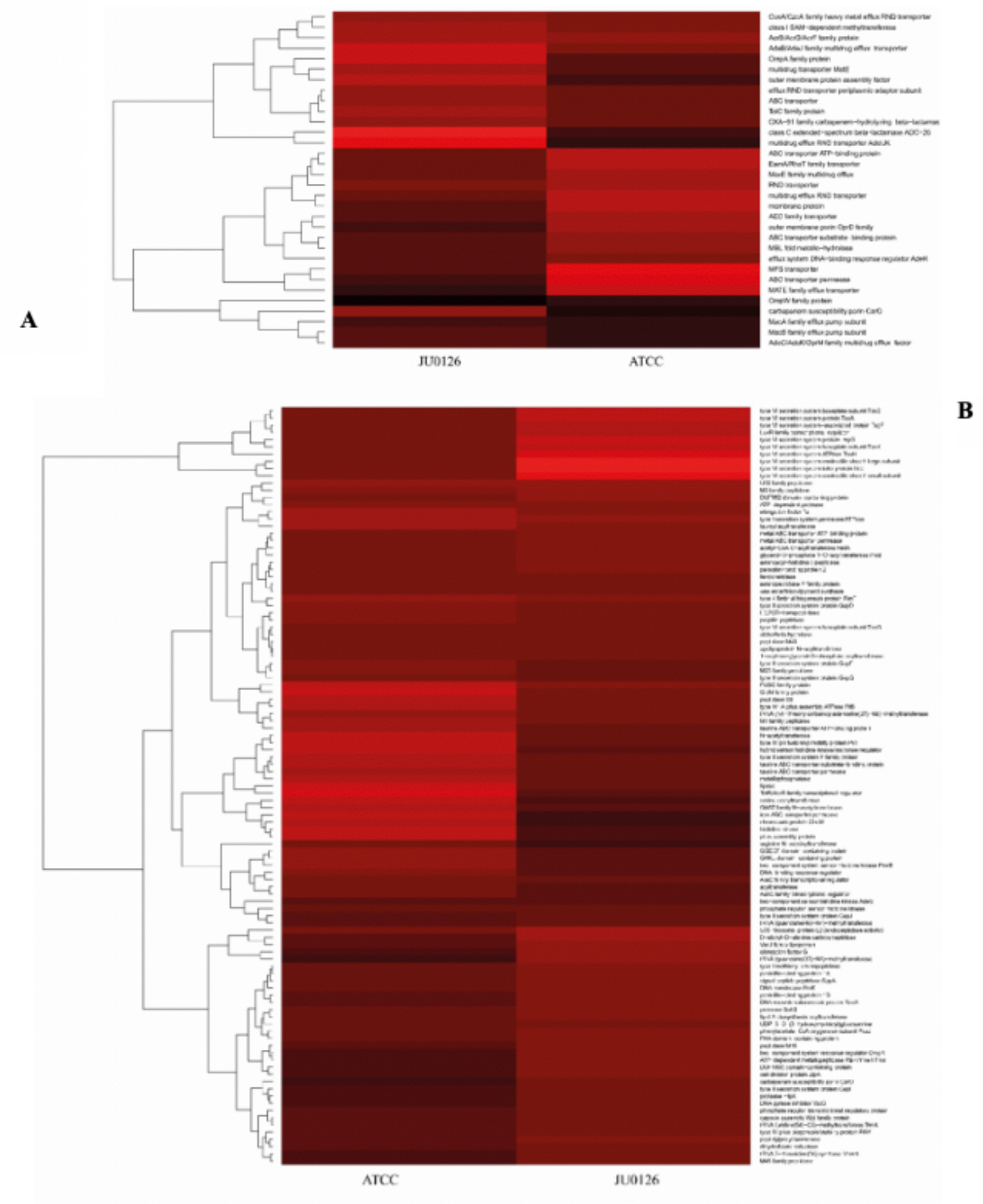

Figure 5

Distribution of genes pertaining to antibiotic resistance and virulence in ATCC 19606 and JU0126 A. baumannii strains 


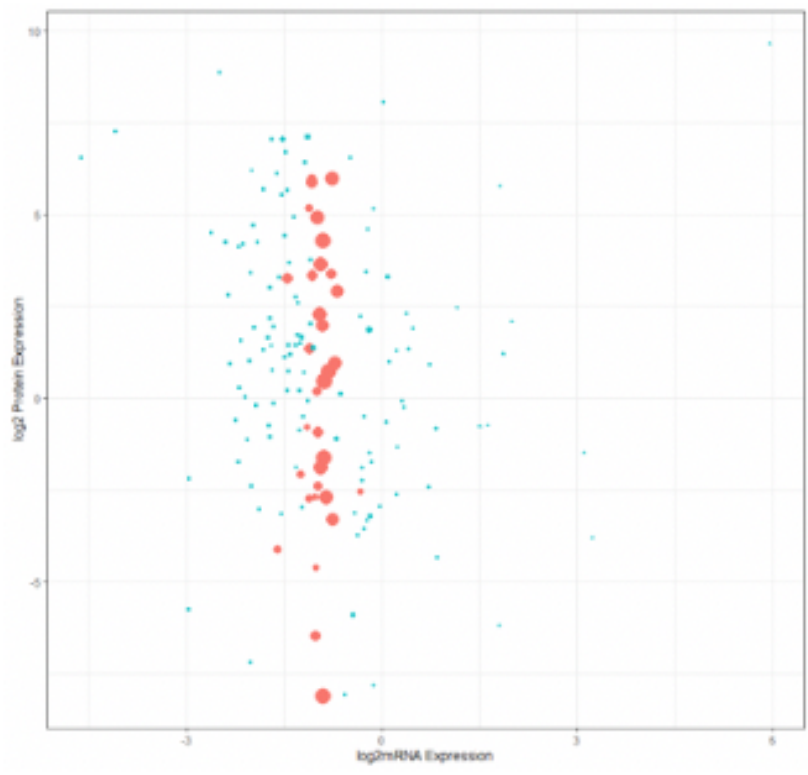

A

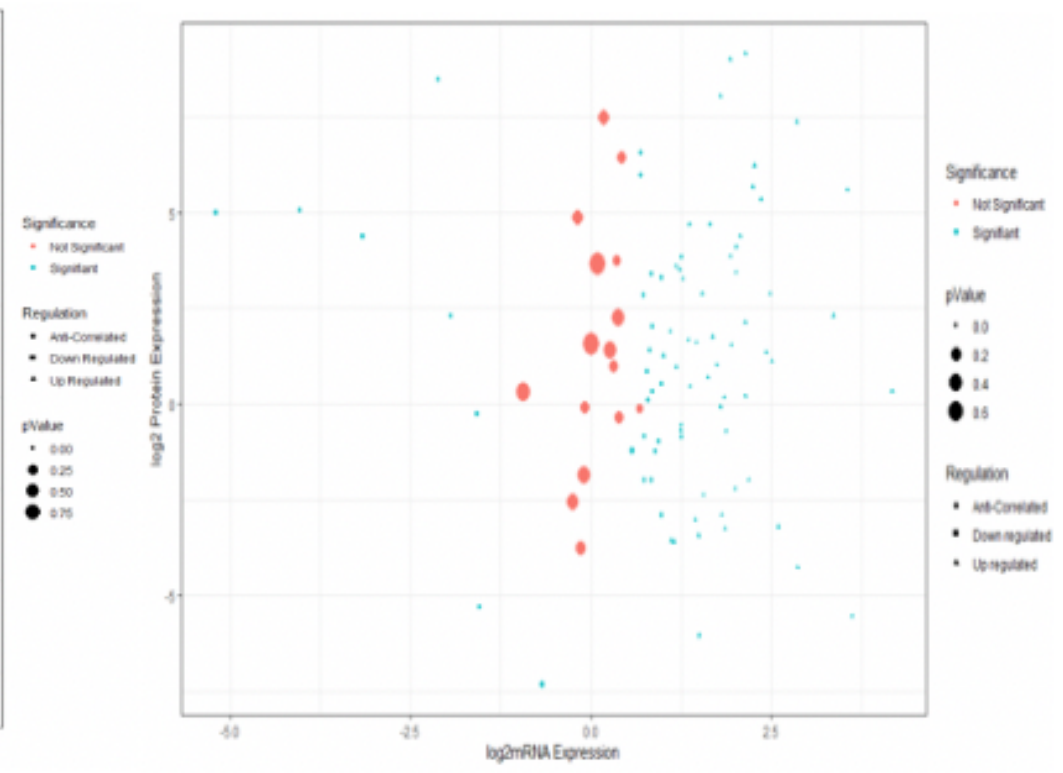

B

\section{Figure 6}

Comparison of whole cell transcriptome and OMV proteome of A. baumannii ATCC 19606 and JU0126 


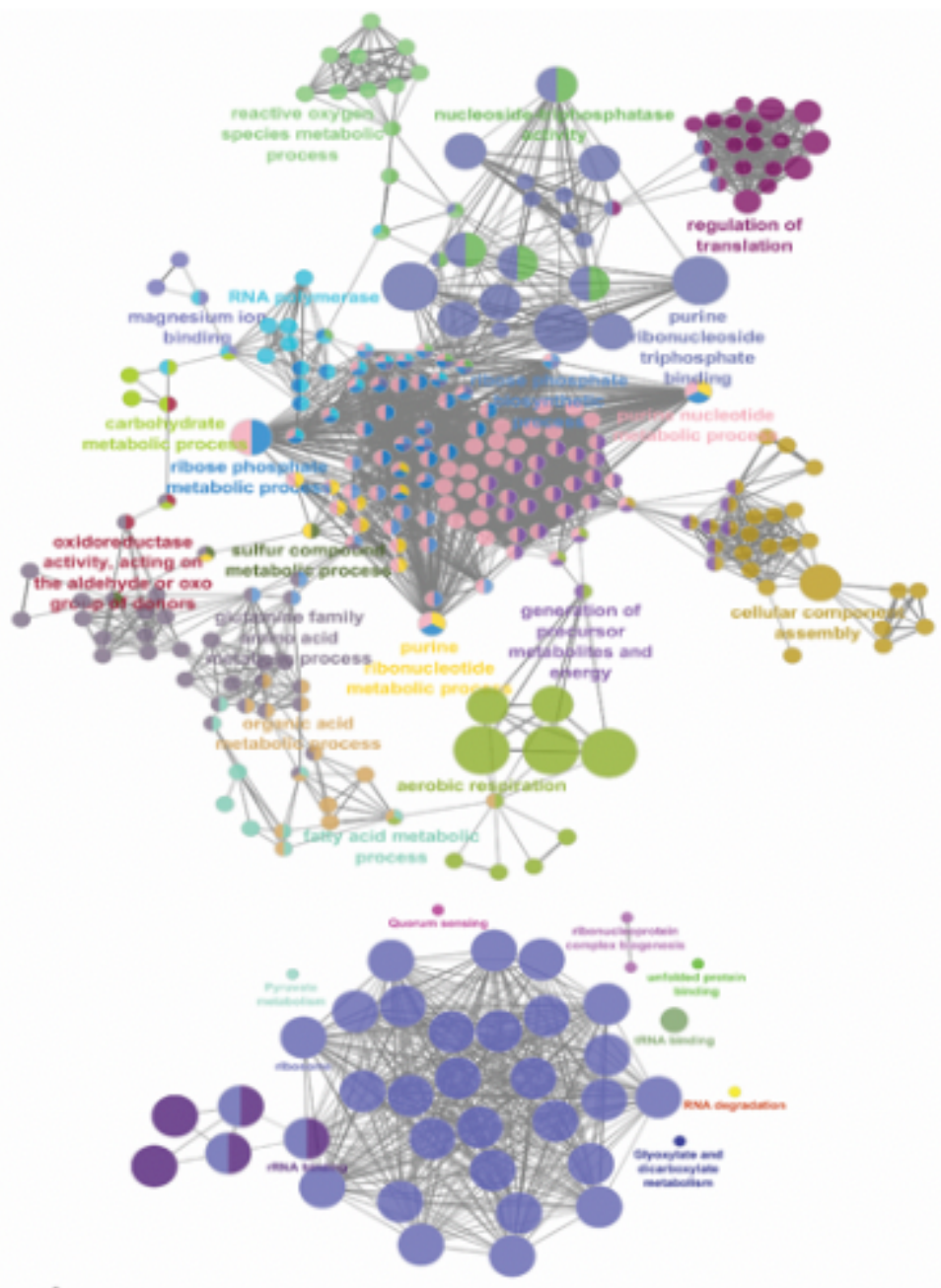

A

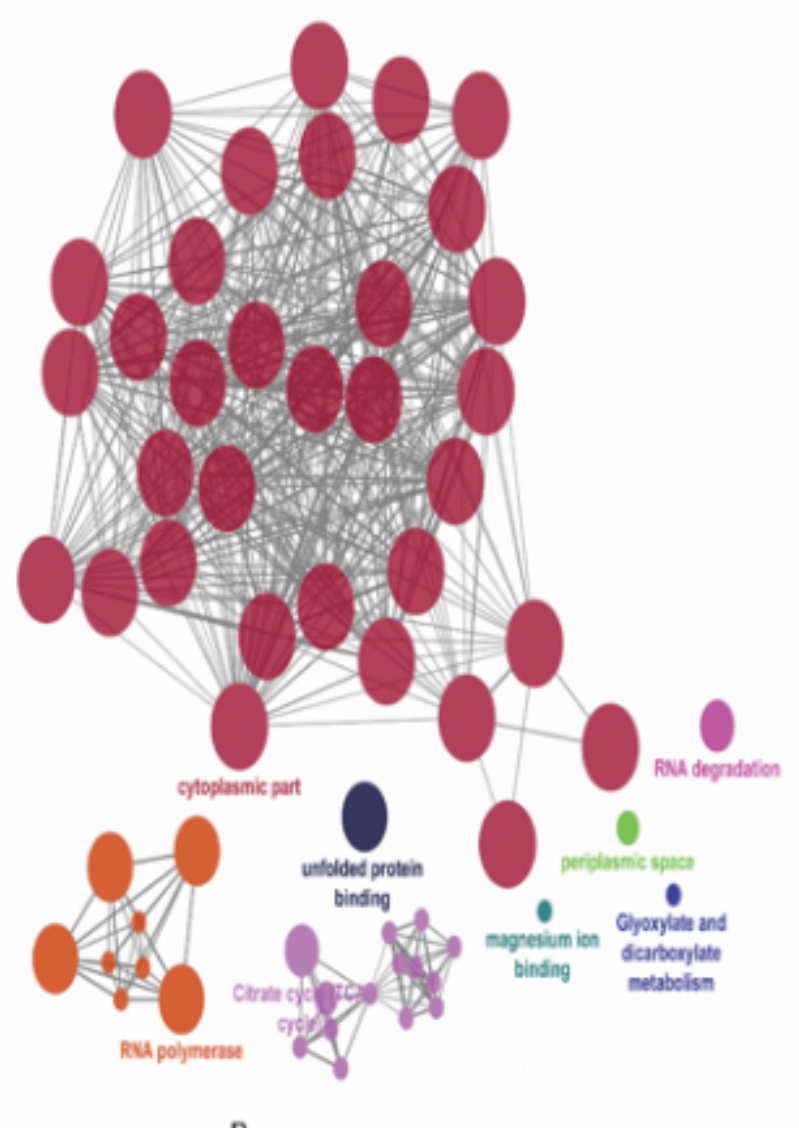

B

Figure 7

Go enrichment analysis and visualization of genes/proteins from both mRNA and OMVs proteome of A. baumannii ATCC 19606 and JU0126 strain using ClueGO/CluePedia plug-in from cytoscape software

\section{Supplementary Files}

This is a list of supplementary files associated with this preprint. Click to download.

- Additionalfile5.xIsx

- Additionalfile1.pdf

- Additionalfile3.xIsx

- Additionalfile4.xlsx

- Additionalfile2.xlsx

- Additionalfile8.pdf

- Additionalfile6.pdf 
- Additionalfile7.docx

Page 33/33 